Revista Internacional de Sociología RIS

vol. 74 (2), e032, abril-junio, 2016, ISSN-L:0034-9712

\section{La clase creativa. Una aproximación a la realidad española}

\author{
José Ángel Bergua Amores \\ Universidad de Zaragoza, España. \\ jabergua@unizar.es \\ DaVID Pac Salas \\ Universidad de Zaragoza, España. \\ davidpac@unizar.es \\ Juan Miguel Báez Melián \\ Universidad de Zaragoza, España. \\ jmbaez@unizar.es \\ Cecilia Serrano Martínez \\ Universidad de Zaragoza, España. \\ cserran@unizar.es
}

Cómo citar este artículo / Citation: Bergua Amores, J. A., D. Pac Salas, J. M. Báez Melián y C. Serrano Martínez. 2016. "La clase creativa. Una aproximación a la realidad española". Revista Internacional de Sociología, 74 (2): e032. Doi: http://dx.doi.org/10.3989/ ris.2016.74.2.032

\section{Resumen}

La noción de clase creativa y su interpretación de la creatividad, elaboradas por Richard Florida (2002), han abierto una importante vía de investigación y controversia en el panorama sociológico internacional. En este artículo aplicamos sus mediciones al caso de España calculando los tres indicadores que construyen la creatividad (tecnología, talento y tolerancia), cada uno de los cuales está compuesto de tres subíndices. Los datos que ofrecemos están desagregados por comunidades autónomas y provincias.
}

\section{Palabras Clave}

Talento; Tecnología; Tolerancia.

\section{Creative class. Approaching Spanish reality}

Copyright: @ 2016 CSIC. Este artículo de acceso abierto distribuido bajo los términos de la licencia Creative Commons Attribution-Non Commercial (by-cn) Spain 3.0.

Recibido: 05/07/2013. Aceptado: 10/04/2015. Publicado on line: 18/05/2016.

\section{Abstract}

The notion of the Creative Class and its interpretation of creativity, developed by Richard Florida (2002), have opened an important avenue of research and controversy in the international sociological scene. In this paper, we apply Florida's measurements to the case of Spain by measuring the three indicators that build creativity (technology, talent and tolerance), each of which is also composed of three sub-indicators. The data are disaggregated by regions and provinces.

\section{KEYWORDS}

Talent; Technology; Tolerance. 


\section{INTRODUCCIÓN}

En los últimos tiempos la creatividad se ha convertido en un importante objeto de reflexión, análisis y controversia. Se habla de ella desde un punto de vista económico (Hawkins 2005), sociológico (Joas 1996; 2012) o psicológico (De Bono 2006; Csíkszentmihályi 1996) y ha entrado en los debates sobre las clases sociales, la ciudad, la industria y la educación entre otros asuntos. Sin embargo, desde que Richard Florida (2010) publicó The Rise of Creative Class en el año 2002, la creatividad ha elevado su popularidad en el ámbito de las ciencias sociales y ha estimulado infinidad de investigaciones ${ }^{1}$. Entre otras, las que él mismo ha realizado o dirigido en relación a Europa (Florida y Tinagli 2004), la competición a nivel mundial por atraer profesionales creativos (Florida 2005a) y la relación de la clase creativa con el desarrollo y cambio de las ciudades y regiones (2005b). Poco después, como suele ocurrir con propuestas arriesgadas o temerarias y de tan alto impacto, aparecieron también las críticas, como las de Peck (2005), Scott y Allen (2006) o Uzzi y Spiro (2005). Igualmente aparecieron trabajos que encontraron útil el indicador para, por ejemplo, mostrar que combinaba bien con las políticas de sostenibilidad urbana (Budd, Lovrich, Pierce, Chamberlain 2008). También algunas decisiones políticas se inspiraron en los trabajos de Florida, como la decisión de la BBC de trasladar ciertas actividades clave al noroeste de Inglaterra (Christopher 2008). En cambio otras políticas, como la aplicada en Escocia, sobrestimaron el ambiente creativo de los territorios para atraer talentos (Houston, Finllay, Harrison y Mason 2008). En España, el primer trabajo sobre este asunto se lo debemos a Casares (2010). También Navarro y Mateos (2010) hacen una aproximación sobre las pautas de localización de la clase creativa en los municipios españoles, si bien utilizan básicamente la variable ocupación.

Dice Hawkins (2005) a propósito de la economía creativa que a finales de los años noventa se hablaba mucho de tecnología, de informática y de tecnologías de la información pero se estaba perdiendo de vista mucho de lo que, en muchos negocios, es el punto de partida: "el hecho de tener ideas". En cierto modo, la innovación, en tanto que "ruptura con las rutinas establecidas en el ámbito productivo" según la formulación ya clásica de Schumpeter, parece referirse a eso. Sin embargo, la innovación se supone que debe tener efectos prácticos, por lo que se distingue de la invención, resultado de una actividad gratuitamente creativa. Por otro lado, suele verse encarnada en una institución o trama institucional, donde pesan más los valores, normas y roles que los más oscuros e imprevisibles elementos profundos de la cultura o los más efímeros de la interacción cotidiana (Fernández Esquino 2012). Además, la innovación está dirigida a "colocar nuevos dominios (ciencia, tecnología e información) bajo el control de la esfera productiva y del mercado, por lo que es evaluada según el cumplimiento de cierta función" (García 2012). En fin, que parece tener un componente instituido mucho más importante que el instituyente. Florida se coloca en una posición ambigua pues apuesta por la creatividad en tanto que ruptura, lo que le llevaría a desmarcarse de la innovación, pero sigue interpretándola en términos de utilidad económica y dependiente de las organizaciones.

En nuestra opinión, la creatividad cuestiona cualquier orden instituido, pues consiste en hacer aparecer algo imprevisto. En este sentido está relacionado con el riesgo (Beck 2002), pues ambos tienen un carácter contingente. En los dos casos, del mismo modo que ocurre en términos más generales con el "acontecimiento", se interrumpe "el curso rutinario y habitual de las cosas" (Badiou 1990:76). Esa interrupción no solo tiene que ver con el límite o fin de algo, sino con la apertura a otra cosa u otros procesos, que para Badiou (1999:317) es, en general, el "exceso del ser" y para nosotros el exceso de lo social. Pero si el riesgo teme ese exceso que desborda el orden instituido y exige precaución, la creatividad lo desea y proyecta "adoración" (Nancy 2010:21-23)2. Hölderlin resumió esta paradójica condición de la modernidad en un hermoso y conocido verso: "Allá donde está el peligro crece también lo que salva"3.

Desde otro punto de vista, la creatividad continúa y profundiza el interés que ya había por el conocimiento (Fumagalli 2010:88-89), resultado de un incremento de las inversiones en este apartado y del abaratamiento de los costes de codificación, transmisión y adquisición de información, lo cual hizo que fuera cada vez más accesible, replicable, móvil e independiente. Pero lo importante es que la explotación económica del conocimiento exigió incluir la base social que lo producía, compuesta por las habilidades lingüísticas y comunicativas depositadas en la propia vida de las gentes (Fumagalli 2010:94-104) e imposibles de desencarnar de ella. Lo mismo ocurrió en el ámbito del consumo de la mano de la publicidad, caracterizada por intentar participar tanto en la opinión y comunicación públicas como en la propia presentación social del individuo (pp. 168-169). La apuesta por la creatividad que realiza el capitalismo contemporáneo, participa de ese mismo impulso pero lo lleva algo más lejos, pues no solo necesita incorporar la sociabilidad y comunicación cotidianas, sino que requiere recursos sociales más básicos y ontológicos como son las "experiencias de autotrascendencia" (Joas 2002:23 y ss.), relacionadas con lo sagrado, y la intervención de lo imaginario (Castoriadis 1989). Ambos recursos tienen que ver con el exceso de lo social que mencionábamos antes.

Aunque Florida tiene en cuenta los estilos de vida, los valores e incluso menciona la imaginación, sus análisis son, en este punto, bastante superficiales ${ }^{4}$. De todas formas, tampoco la sociología ha escapa- 
do a este problema. Las dos tradiciones dominantes en la reflexión sobre la acción social, la que enfatiza la acción normativamente orientada y la que subraya su carácter racional, son incapaces de dar cuenta de la creatividad (Joas 1996:4-5). Más allá de la acción social, en el ámbito de la producción cultural, la sociología tampoco ha sabido tratar la creatividad, pues siempre ha preferido prestar atención a lo que la limita, encauza e institucionaliza (Noya 2010). Por ello, nuestra intención en este artículo es aplicar la medida cuantitativa que utilizó en Estados Unidos, primero, y luego en Europa, más consistente que la cualitativa y todavía no realizada en España desde un punto de vista sociológico. Su indicador de creatividad combina los índices de tecnología, talento y tolerancia que, a su vez, están compuestos por otros tres subíndices. La tecnología incluye subíndices relativos a I+D, Innovación y Alta Tecnología. Por lo que respecta al talento, sus componentes son el volumen de las clases creativas, el número de titulados y la cantidad de investigadores. Finalmente, la tolerancia, fue medida en Estados Unidos a partir de la cantidad de extranjeros, bohemios y homosexuales, mientras que para el caso de Europa tuvo en cuenta las actitudes hacia las minorías, la autoexpresión y el índice de valores.

El objeto de este artículo es aplicar la medición cuantitativa a España desagregando los datos por comunidades Autónomas y Provincias. La principal fuente de información nos la proporciona el Censo de Población de 2011. De todas formas haremos alguna referencia a otros datos que nos permitan construir e interpretar los diferentes indicadores e índices de creatividad utilizados.

Para elaborar el índice de creatividad hemos procedido de la manera que sigue. Primero, obtuvimos información sobre los subíndices que incorporan la tecnología, el talento y la tolerancia. Esta fue medida, como en Estados Unidos, a partir del número de extranjeros, homosexuales y bohemios, pues los datos de la encuesta mundial de valores que utilizó Florida en la investigación de Europa no están desagregados por regiones y en las bases de datos del CIS, CIRES y otras también falta esa información. Para cada uno de los subíndices elaboramos una clasificación de comunidades autónomas y provincias (en este segundo caso, cuando ha sido posible) puntuando con 100 a los territorios que mejores números obtuvieron y otorgando al resto puntuaciones proporcionales en relación a ese techo. Después de obtener esos subíndices, calculamos los índices de tecnología, talento y tolerancia averiguando la media de las puntuaciones obtenidas por cada territorio. Finalmente, el índice de creatividad de cada territorio se elaboró sumando las puntuaciones obtenidas en las tres T (tecnología, talento y tolerancia) y obteniendo la media aritmética. Por lo tanto, la clasificación final de creatividad está calculado a partir de un máximo de 100 que habría obtenido el territorio que hubiera sido primero en todos los índices y subíndices.

\section{TeCnología}

Un objetivo presente en toda la historia del pensamiento económico ha sido averiguar las causas del desarrollo y su medición. Tanto es así que en los orígenes de la ciencia económica como tal, es decir, en los textos de Adam Smith, ya se hablaba del crecimiento económico como uno de los objetivos fundamentales de la política económica. Sin embargo, el concepto ha variado considerablemente desde los tiempos de la primera revolución industrial.

Hasta mediados del siglo $X X$ se hablaba simplemente de crecimiento y su medición era el PNB o el PIB (Payne y Philips 2011). El objetivo político era que estos indicadores crecieran lo máximo posible, por encima de las tasas de crecimiento de la población. Por otro lado, el sector clave era el industrial, por lo que un país industrializado era equivalente a un país desarrollado.

En las siguientes décadas aparecieron las limitaciones del desarrollo así definido. Había actividades necesarias para el buen funcionamiento del sistema económico que no estaban contabilizadas en el PNB, por ejemplo los servicios domésticos o el cuidado de las personas dependientes (niños, ancianos y enfermos). Por otra parte, determinadas actividades productivas tenían un coste social en términos medioambientales, que tampoco se estaba teniendo en cuenta. Se empezó así a hablar de desarrollo (más allá del simple crecimiento productivo) y, más concretamente, de desarrollo sostenible, es decir, un desarrollo que no dilapidara los recursos naturales.

Por otro lado, las dificultades de los países del Tercer Mundo para encontrar la senda del desarrollo incentivó la búsqueda de ideas que hicieran más realista y operativo el concepto. Amartya Sen, por ejemplo, planteó los contrastes habidos entre los niveles de vida valorados en función de la renta per cápita y los valorados en función de la capacidad para sobrevivir hasta edades avanzadas, demostrando que las causas de dichos contrastes se encontraban en "las instituciones sociales y las relaciones en el seno de la comunidad, como la cobertura médica, la sanidad pública, la educación escolar, el orden público, el grado de violencia, etc." (2000). Surgió así el concepto de "desarrollo humano" y, ligado a él, el Índice de Desarrollo Humano, un índice calculado por el PNUD cuyo cómputo está basado en la esperanza de vida, el sistema educativo y el nivel de renta.

Por último, el considerable desarrollo de las tecnologías de la información y comunicación, que ha tenido lugar durante las últimas décadas, han vuelto de nuevo a cuestionar los conceptos básicos. La idea fundamental es que los inputs principales ya no son únicamente la tierra, el trabajo y el capital, ya que la información y el conocimiento han pasado a considerarse factores productivos esenciales. El conocimiento como factor clave, que solo puede 
adquirirse mediante la escolarización, es fundamentalmente diferente de los tradicionales recursos claves de los economistas pues no conoce fronteras, es rápido, su transporte barato y, por definición, cambiante (Drucker 1994).

Precisamente, para Florida (2010), dos de los elementos principales de la creatividad son la información y el conocimiento. El resultado de esa creatividad es la innovación, ya sea en forma de un nuevo artilugio tecnológico o una nueva forma organizativa empresarial. Estados Unidos es el líder mundial de la economía creativa y especialmente en su aspecto tecnológico. Estos son algunos de los datos que lo demuestran:

- Teniendo en cuenta la inflación, el gasto en I+D creció en un $800 \%$ durante el periodo 19532000.

- La cantidad de patentes concedidas se ha multiplicado por seis durante el periodo 1900-1999.

- La población activa dedicada a la creatividad técnica (científicos e ingenieros) se ha octuplicado desde mediados de siglo.

- La cantidad de personas que vivían de la creatividad artística y cultural eran aproximadamente 250 por cada 100.000 estadounidenses. Dicha cifra pasó a ser de 900 personas en 1999.

En ese mismo trabajo, Florida aborda los aspectos relacionados con la tecnología mediante la construcción de dos índices para las distintas regiones de Estados Unidos. Por un lado, el tecnológico, que es la combinación de dos factores: la producción industrial de alta tecnología, como porcentaje de la producción de alta tecnología estadounidense, y el porcentaje de la producción regional procedente de industrias de alta tecnología, en comparación con el porcentaje nacional. La utilización de estos dos factores se basa en que el primero favorece a las áreas metropolitanas de gran tamaño, mientras que el segundo a las regiones más pequeñas con grandes sectores industriales. El otro índice empleado fue el de innovación, basado en las patentes per cápita de cada región.

En un trabajo posterior (Florida y Tinagli 2004), en el que este mismo autor realiza un estudio similar para 14 países de la UE y Estados Unidos, construye un solo índice tecnológico basado en tres medidas: el gasto en I+D como porcentaje del PIB, el número de patentes por millón de habitantes, y el número de patentes en alta tecnología por millón de habitantes en campos tales como la biotecnología, tecnología de la información, farmacia e industria aeroespacial (Cuadro I).

Lo primero que resalta de este cuadro es la gran ventaja tecnológica de Estados Unidos con respecto a los países europeos. Dicho país obtiene más de diez puntos sobre el segundo clasificado. La ventaja se plasma en las patentes y, especialmente, en la alta tecnología, lo que debe ser un
Cuadro I.

El Índice Tecnológico para Europa y Estados Unidos

\begin{tabular}{|l|c|c|c|c|}
\hline & Total & I+D & Patentes & Alta tecnología \\
\hline Estados Unidos & 40,62 & 10,62 & 15,00 & 15,00 \\
\hline Suecia & 29,58 & 15,00 & 9,33 & 5,25 \\
\hline Finlandia & 25,91 & 13,38 & 6,14 & 6,39 \\
\hline Alemania & 18,86 & 9,97 & 6,33 & 2,56 \\
\hline Dinamarca & 15,95 & 8,39 & 4,48 & 3,08 \\
\hline Holanda & 15,78 & 7,86 & 4,43 & 3,49 \\
\hline Bélgica & 14,50 & 8,03 & 4,19 & 2,28 \\
\hline Francia & 14,46 & 8,80 & 3,29 & 2,37 \\
\hline Reino Unido & 13,57 & 7,58 & 3,43 & 2,56 \\
\hline Austria & 11,89 & 7,22 & 3,67 & 1,00 \\
\hline Irlanda & 8,37 & 5,64 & 2,05 & 0,68 \\
\hline Italia & 6,49 & 4,22 & 1,52 & 0,75 \\
\hline España & 4,21 & 3,65 & 0,38 & 0,18 \\
\hline Portugal & 3,23 & 3,16 & 0,05 & 0,02 \\
\hline Grecia & 2,26 & 2,07 & 0,10 & 0,09 \\
\hline
\end{tabular}

Fuente: Florida y Tinagli 2004. Aunque las cifras totales las hemos obtenido sumando los tres subíndices.

reflejo de que la última revolución tecnológica ha tenido lugar con antelación y con mayor fortaleza en Estados Unidos que en Europa. Por otra parte, en los países europeos se observa una distribución regional del Índice. En primer lugar, tenemos a los países escandinavos, Suecia y Finlandia, con una puntuación total entre veinte y treinta puntos, destacando el primero de ellos en el gasto en investigación y desarrollo. En segundo lugar tenemos a los países del Centro de Europa y el Reino Unido, que oscilan entre diez y veinte puntos globales. Luego tenemos el caso de Irlanda, 8,37 puntos, y ocupando los últimos puestos de la clasificación se sitúan los países del Sur de Europa: Italia, España, Portugal y Grecia.

En nuestro caso hemos construido el índice tecnológico a partir de tres subíndices que lo componen: gasto en I+D, innovación (patentes concedidas por millón de habitantes) y Alta Tecnología (patentes en Alta Tecnología concedidas por millón de habitantes). En el Cuadro II tenemos los resultados de dicho índice para las comunidades españolas. Como ya avanzamos, las autonomías puntúan en cada subíndice a partir de un máximo de 100 obtenido por la primera; luego, hemos sumado los tres subíndices, y finalmente hemos obtenido la media para obtener la clasificación final (quinta columna). Como vemos en el mencionado cuadro, los tres primeros lugares están ocupados por las comunidades que tienen el mejor resultado en alguno de los subíndices. Efectivamente, Madrid, Navarra y País Vasco obtienen el mejor resultado en los subíndices de Alta Tecnología, Patentes e I+D, respectivamente. Aragón se sitúa ligeramente por debajo del País Vasco, aunque con una cifra baja en I+D. Los prime- 
ros lugares los cierra Cataluña, con malos resultados en alta tecnología. En realidad, en este subíndice los números tan bajos se deben al excelente resultado de la Comunidad de Madrid, que obtuvo 49,77 patentes en alta tecnología durante el año 2011, muy superior a la media de ese año: 15,70.

\section{Cuadro II.}

Índice tecnológico por Comunidades Autónomas para el año 2011.

\begin{tabular}{|l|c|c|c|c|}
\hline & Innovación & I+D & $\begin{array}{c}\text { Alta } \\
\text { Tecnología }\end{array}$ & Clasificación \\
\hline Madrid & 67,49 & 88,56 & 100,00 & 85,35 \\
\hline Navarra & 100,00 & 98,14 & 44,05 & 80,73 \\
\hline País Vasco & 65,93 & 100,00 & 40,48 & 68,80 \\
\hline Aragón & 92,75 & 44,22 & 64,26 & 67,08 \\
\hline Cataluña & 50,18 & 72,38 & 42,84 & 55,13 \\
\hline Galicia & 40,86 & 43,95 & 44,94 & 43,25 \\
\hline Cantabria & 39,72 & 52,36 & 37,36 & 43,14 \\
\hline Comunidad & 41,55 & 48,35 & 26,51 & 38,80 \\
\hline Valenciana & 34,40 & 53,13 & 25,26 & 37,60 \\
\hline Andalucía & 40,91 & 45,17 & 16,82 & 34,30 \\
\hline Asturias & 41,28 & 48,23 & 12,52 & 34,01 \\
\hline La Rioja & 24,18 & 48,58 & 18,97 & 30,58 \\
\hline Castilla-León & 29,69 & 40,00 & 19,25 & 29,65 \\
\hline Murcia & 18,56 & 38,10 & 12,74 & 23,14 \\
\hline Extremadura & 22,91 & 31,11 & 12,42 & 22,15 \\
\hline Castilla-La Mancha & 15,69 & 27,56 & 6,78 & 16,68 \\
\hline Canarias & 8,80 & 17,13 & 11,00 & 12,31 \\
\hline Baleares & & &
\end{tabular}

Se puede apreciar, por tanto, el predominio de las regiones del noroeste de la Península en el Índice Tecnológico. Por otra parte, los peores resultados son obtenidos por comunidades situadas en el sur, destacando los dos archipiélagos, caracterizados por tener unas economías basadas en el turismo.

En el Cuadro III tenemos de nuevo la clasificación obtenida para el año 2011 (columna 1), la obtenida con las mismas variables y fuentes para el año 2001 (columna 2) y la obtenida por Casares et al. (2012), esta última con datos de los años 20012004. La primera conclusión que se deriva de la observación de este cuadro es que la Comunidad de Madrid es la que tiene el predominio en la cuestión tecnológica en España. Aunque dicho predominio parece haberse reducido durante la primera década de este siglo, como lo demuestra el menor valor del índice en el año 2011 (85,35 frente a 93,84 en el año 2001), cuando se ha utilizado la misma metodología y la misma fuente de datos para construirlo. Esta reducción en el predominio tecnológico también se refleja en la reducción del cociente entre el valor del índice y la media de las 17 comunidades: de 2,2 a 2,0 .

Sin embargo, dicha reducción es bastante más significativa si utilizamos como referencia inicial los
Cuadro III.

Comparación entre las diferentes clasificaciones.

\begin{tabular}{|l|c|c|c|}
\hline & $\begin{array}{c}\text { Año } \\
2011\end{array}$ & $\begin{array}{c}\text { Año } \\
2001\end{array}$ & $\begin{array}{c}\text { Casares } \\
(2001-2004)\end{array}$ \\
\hline Madrid & 1 & 1 & 1 \\
\hline Navarra & 2 & 2 & 3 \\
\hline País Vasco & 3 & 4 & 4 \\
\hline Aragón & 4 & 5 & 5 \\
\hline Cataluña & 5 & 3 & 2 \\
\hline Galicia & 6 & 8 & 15 \\
\hline Cantabria & 7 & 10 & 8 \\
\hline Comunidad Valenciana & 8 & 6 & 6 \\
\hline Andalucía & 9 & 11 & 12 \\
\hline Asturias & 10 & 7 & 10 \\
\hline La Rioja & 11 & 12 & 9 \\
\hline Castilla-León & 12 & 9 & 14 \\
\hline Murcia & 13 & 13 & 13 \\
\hline Extremadura & 14 & 14 & 17 \\
\hline Castilla-La Mancha & 15 & 17 & 16 \\
\hline Canarias & 16 & 16 & 11 \\
\hline Baleares & 17 & 15 & 7 \\
\hline
\end{tabular}

Fuente: INE y Casares (2012). Elaboración propia.

resultados del trabajo de Casares et al. (2012): de 2,7 a 2,0. Téngase en cuenta que estos autores sustituyen nuestro subíndice $\mathrm{I}+\mathrm{D}$ (gasto en $\mathrm{I}+\mathrm{D}$ como porcentaje del PIB) por el porcentaje de hogares con conexión a Internet y el porcentaje de hogares con ordenador (además de la diferente metodología para la propia construcción del índice).

Otra cuestión que cabe destacar del Cuadro III es que en las tres calificaciones, los cinco primeros puestos están ocupados por las mismas comunidades: Madrid, Navarra, País Vasco, Aragón y Cataluña, pero no en las mismas posiciones. Parece que durante la primera década de este siglo ha habido una mejora de Aragón a costa de Cataluña, que ha quedado relegada al quinto lugar. La reducción en el valor del índice de esta comunidad, según nuestros datos, ha sido significativa: de 72,51 en el año 2001 a 55,13 en el 2011, aunque también hay que decir que Aragón ha pasado de 57,71 a 67,08.

Por debajo del puesto ha habido otras mejoras por parte de las siguientes comunidades: Galicia, Cantabria, Comunidad Valenciana y Andalucía.

Por último, también queremos resaltar las similitudes entre los resultados del trabajo de Casares et al. (2012) y los nuestros del año 2001, al menos en los cinco primeros puestos. En el resto de la clasificación hay algunas diferencias que resultan significativas. Por ejemplo, los casos de Castilla-León y Canarias, con cinco puestos de diferencia y, sobre todo, los de Galicia (siete puestos) y Baleares (ocho).

En el Cuadro III presentamos los resultados de aplicar nuestro índice tecnológico para las provin- 
cias españolas, también para el año 2011. En este caso, dada la imposibilidad de conseguir los datos del gasto en I+D desagregados por provincia, hemos tenido que construir el mismo únicamente con los otros dos subíndices, esto es, con el de Innovación y Alta Tecnología. La centralización de la actividad innovadora en Zaragoza, por un lado, y la supresión del subíndice I+D (en el que, como vimos antes, la comunidad aragonesa obtiene la peor nota), colocan a la provincia de Zaragoza a la cabeza de la clasificación de nuestro índice tecnológico (cuarta columna). El segundo lugar lo ocupa la comunidad de Madrid, gracias a su primer lugar en el subíndice de alta tecnología, tal y como ocurría en la clasificación por comunidades autónomas.

El resto de los primeros lugares están ocupados principalmente por regiones del Noroeste español, como pasaba con las comunidades autónomas. Sin embargo, entre las diez primeras están La Coruña (cuarto lugar) y Sevilla (noveno), la primera debida a su buena puntuación en alta tecnología, y la segunda por tener una puntuación aceptable en ambos subíndices. Es de destacar también la presencia de las tres provincias vascas en los diez primeros lugares.

En cuanto a los últimos lugares cabe destacar, por un lado, la última posición ocupada por Teruel, cuando Aragón se sitúa en el cuarto lugar, como comunidad autónoma; por otro lado, también sorprende la presencia de Lugo entre los diez últimos, cuando Galicia es la sexta entre las comunidades autónomas.

Para comprobar de nuevo qué es lo que ha pasado con nuestro índice tecnológico durante la primera década del siglo XXI, hemos construido el Cuadro IV, donde tenemos los puestos ocupados por cada una de las provincias en los años 2011 (segunda columna) y 2001 (tercera columna). Lo primero que queremos resaltar de este cuadro es el desplazamiento de Madrid del primer puesto, por parte de Zaragoza, lo que explica también en parte el ascenso de la comunidad aragonesa.

Por otra parte, las provincias que ocupan los diez primeros lugares en el año 2011, siete de ellas ya los ocupaban en el año 2001: Zaragoza, Madrid, Navarra, Álava, Barcelona, Guipúzcoa y Valencia. No obstante, hay tres provincias que experimentaron ascensos, dos de ellos considerables, La Coruña (del 27 al 4) y Sevilla (del 30 al 9) (lo que puede explicar en cierta medida los progresos de Galicia y Andalucía, respectivamente); mientras que Vizcaya lo hizo en menor medida (del 13 al 8).

En cuanto a las diez últimas posiciones, solo dos provincias no estaban en esos lugares en el año 2001: Las Palmas y Cáceres, con un considerable de descenso en ambos casos.
Cuadro IV.

Índice tecnológico por provincias para el año 2011.

\begin{tabular}{|c|c|c|c|}
\hline & Innovación & $\begin{array}{c}\text { Alta } \\
\text { Tecnología }\end{array}$ & Clasificación \\
\hline Zaragoza & 100,00 & 84,50 & 92,25 \\
\hline Madrid & 56,88 & 100,00 & 78,44 \\
\hline Navarra & 84,28 & 44,05 & 64,16 \\
\hline La Coruña & 41,42 & 75,78 & 58,60 \\
\hline Álava & 47,52 & 56,41 & 51,96 \\
\hline Barcelona & 47,99 & 55,04 & 51,52 \\
\hline Guipúzcoa & 57,41 & 42,59 & 50,00 \\
\hline Vizcaya & 56,66 & 34,76 & 45,71 \\
\hline Sevilla & 43,25 & 43,83 & 43,54 \\
\hline Valencia & 38,08 & 36,89 & 37,48 \\
\hline Pontevedra & 39,91 & 33,69 & 36,80 \\
\hline Cantabria & 32,61 & 37,36 & 34,99 \\
\hline Albacete & 30,37 & 35,05 & 32,71 \\
\hline Valladolid & 28,62 & 33,97 & 31,29 \\
\hline Granada & 35,29 & 26,18 & 30,73 \\
\hline Burgos & 28,61 & 26,96 & 27,78 \\
\hline Málaga & 21,74 & 32,89 & 27,32 \\
\hline Almería & 39,88 & 11,69 & 25,79 \\
\hline Asturias & 34,47 & 16,82 & 25,65 \\
\hline Castellón & 40,99 & 10,14 & 25,57 \\
\hline Huelva & 24,45 & 23,22 & 23,83 \\
\hline La Rioja & 34,79 & 12,52 & 23,65 \\
\hline Huesca & 29,19 & 17,77 & 23,48 \\
\hline Alicante & 28,85 & 17,40 & 23,13 \\
\hline Murcia & 25,02 & 19,25 & 22,14 \\
\hline Soria & 21,45 & 21,22 & 21,34 \\
\hline Lerida & 31,35 & 9,19 & 20,27 \\
\hline León & 23,67 & 16,29 & 19,98 \\
\hline Jaén & 24,34 & 15,05 & 19,69 \\
\hline Guadalajara & 21,79 & 15,67 & 18,73 \\
\hline Salamanca & 14,50 & 22,95 & 18,72 \\
\hline Badajoz & 19,84 & 17,45 & 18,64 \\
\hline Gerona & 30,48 & 5,36 & 17,92 \\
\hline Orense & 21,61 & 12,21 & 16,91 \\
\hline Tarragona & 20,17 & 12,47 & 16,32 \\
\hline Cádiz & 19,62 & 11,32 & 15,47 \\
\hline Toledo & 15,15 & 11,42 & 13,28 \\
\hline Santa Cruz de Tenerife & 15,36 & 8,11 & 11,74 \\
\hline Córdoba & 13,93 & 7,51 & 10,72 \\
\hline Islas Baleares & 7,42 & 11,00 & 9,21 \\
\hline Zamora & 7,93 & 10,46 & 9,20 \\
\hline Ciudad Real & 17,37 & 0,00 & 8,68 \\
\hline Las Palmas & 11,72 & 5,57 & 8,65 \\
\hline Lugo & 8,74 & 5,76 & 7,25 \\
\hline Cuenca & 14,13 & 0,00 & 7,06 \\
\hline Cáceres & 8,61 & 4,87 & 6,74 \\
\hline Segovia & 12,44 & 0,00 & 6,22 \\
\hline Palencia & 11,89 & 0,00 & 5,94 \\
\hline Ávila & 8,86 & 0,00 & 4,43 \\
\hline Teruel & 7,07 & 0,00 & 3,54 \\
\hline
\end{tabular}




\section{TALENTO}

El talento constituye, sin duda alguna, un aspecto básico en los trabajos de Florida (2010). Para darle operatividad al concepto utiliza tres indicadores básicos: el Índice de Ocupaciones Creativas, el Índice de Capital Humano y el Índice de Talento Científico.

La ocupación como indicador de clase social ${ }^{5}$ no es muy original, ya que en la literatura sociológica y económica viene usándose desde hace varias décadas. Sobre todo vinculando la ocupación al nivel de la titulación requerida para su desempeño. Florida (2010:418 y ss.) utiliza las siguientes categorías laborales en la definición de las clases sociales básicas (Cuadro V).

\section{Cuadro V.}

Clasificación de categorías laborales creativas según Florida.

\begin{tabular}{|l|}
\hline Núcleo supercreativo \\
\hline Informática y matemáticas \\
\hline Arquitectura y las distintas ingenierías \\
\hline Ciencias sociales, físicas y de la vida \\
\hline Educación, enseñanza y lectura \\
\hline Arte, diseño, entretenimiento, deporte y medios de comunicación \\
\hline Resto de profesionales creativos \\
\hline Puestos de alta dirección \\
\hline Empresa y finanzas \\
\hline Sector jurídico \\
\hline Profesiones sanitarias y técnicas \\
\hline Ventas de alto nivel y gestión de ventas \\
\hline
\end{tabular}

Según sus aproximaciones, en 1999 había en EE. UU. un 30,8 \% de ocupaciones creativas que absorbían hasta la mitad de los ingresos salariales, por encima de la manufactura y servicios juntos ${ }^{6}$. El núcleo supercreativo constituía el $11,7 \%$. Aunque a principios de siglo XX representaban únicamente un $10 \%$ de los trabajos, a partir de 1950 es cuando empiezan a tener un pequeño crecimiento hasta la década de los ochenta. La expansión de estas ocupaciones se producirá fundamentalmente en la década de los 90 hasta alcanzar a casi un tercio de las ocupaciones (Florida 2010:212-123).

En su investigación europea, Florida y Tinagli (2004:14) atienden a dos indicadores sobre talento: porcentaje de empleados en ocupaciones creativas con respecto al total de ocupados, y la variación anual de esas ocupaciones desde 1995-2000. Señalan que las clases creativas suponen más de un cuarto de los ocupados en cinco países: Bélgica $(29,97 \%)$, Holanda (29,5\%), Finlandia (28,6\%), Reino Unido (26,7 \%) e Irlanda (26\%); ocupan los primeros lugares en la clasificación europea. Es decir, porcentajes muy similares al $30 \%$ que hemos visto en EE. UU. En el polo opuesto, los países con menores ocupaciones creativas son Italia y Portugal, con porcentajes por debajo del $15 \%$. España ocupa una posición intermedia con un 19,8 $\%$. Por último, en relación a la evolución destaca Irlanda con un incremento de las ocupaciones creativas del $7 \%$ anual desde 1995.

Según nuestros datos, en el año 2011 hay en España un 21,02 \% de ocupaciones que podemos denominar creativas ${ }^{7}$. Es decir, uno de cada cuatro ocupados tienen componentes de creatividad en las tareas que realizan. Entre las principales ocupaciones creativas podemos establecer dos tipos de actividades: tareas propias de dirección y gerencia empresarial (casi 4 de cada 10 trabajos creativos) y actividades vinculadas a profesiones asociadas a titulaciones universitarias (más del $55 \%$ ). Si nos centramos en la desagregación de las ocupaciones supercreativas, ocupan el primer puesto en la clasificación los ocupados en la dirección de empresas de más de 10 asalariados y los profesores de secundaria y universitaria que suponen casi la mitad de estas ocupaciones (46, $5 \%$ ).

Las comunidades autónomas con un porcentaje de ocupados en trabajos creativos superiores a la media española son (Cuadro VI): Madrid, País Vasco, Cataluña, Navarra y Aragón. Sobre todas ellas destaca Madrid con más de 6 puntos por encima de la media española. En el otro extremo nos encontramos a Castilla-León, La Rioja y Castilla-La Mancha con un porcentaje de ocupaciones creativas por debajo del $18 \%$.

Cuadro Vl.

Clasificación de CC. AA. por Ocupaciones Creativas (2011).

\begin{tabular}{|l|c|c|}
\hline Madrid & 27,09 & 100,0 \\
\hline País Vasco & 22,50 & 83,1 \\
\hline Cataluña & 22,31 & 82,4 \\
\hline Navarra & 20,68 & 76,3 \\
\hline Comunidad Valenciana & 20,05 & 74,0 \\
\hline Andalucía & 19,83 & 73,2 \\
\hline Islas Baleares & 19,52 & 72,1 \\
\hline Asturias & 19,45 & 71,8 \\
\hline Cantabria & 19,15 & 70,7 \\
\hline Extremadura & 18,91 & 69,8 \\
\hline Aragón & 18,66 & 68,9 \\
\hline Galicia & 18,18 & 67,1 \\
\hline Murcia & 18,08 & 66,7 \\
\hline Canarias & 18,05 & 66,6 \\
\hline Castilla-León & 17,85 & 65,9 \\
\hline La Rioja & 17,12 & 63,2 \\
\hline Castilla - La Mancha & 16,82 & 62,1 \\
\hline
\end{tabular}

Fuente: Censo de 2011. Elaboración propia. 
Todavía nos encontramos con diferencias más significativas si analizamos las ocupaciones a nivel provincial (Cuadro VII). Entre los 10 primeros puestos nos encontramos a Madrid, que ocupa la primera posición con más de cada cuatro trabajadores en ocupaciones creativas (27,1\%). Barcelona, Vizcaya, Sevilla, Granada, Guipúzcoa, Valencia, Valladolid, Navarra, Álava y Salamanca.

\section{Cuadro VII.}

Clasificación de provincias por Ocupaciones Creativas (2011)

\begin{tabular}{|l|c|}
\hline \multicolumn{2}{|c|}{ Las 10 primeras } \\
\hline Madrid & 27,1 \\
\hline Barcelona & 23,7 \\
\hline Vizcaya & 23,5 \\
\hline Sevilla & 22,8 \\
\hline Granada & 22,7 \\
\hline Guipúzcoa & 22,0 \\
\hline Valencia & 21,8 \\
\hline Valladolid & 21,2 \\
\hline Navarra & 20,7 \\
\hline Álava & 20,3 \\
\hline & \\
\hline León & 16,3 \\
\hline Segovia & 16,2 \\
\hline Soria 10 últimas & 16,1 \\
\hline Orense & 16,1 \\
\hline Palencia & 15,9 \\
\hline Zamora & 15,6 \\
\hline Lugo & 15,2 \\
\hline Cuenca & 14,8 \\
\hline Toledo & 14,8 \\
\hline Teruel & 14,4 \\
\hline
\end{tabular}

Fuente: Censo de 2011. Elaboración propia.

En el otro extremo, nos encontramos a Teruel que ocupa el último lugar con un $14,4 \%$ y hay otras provincias con escasas ocupaciones creativas: Toledo, Cuenca, Lugo, Zamora, Palencia, Orense, Soria, Segovia-León, donde las ocupaciones creativas suponen menos de $17 \%$ sobre el total de ocupados.

El segundo indicador de talento lo constituye el Índice de Capital Humano, que tiene en cuenta el porcentaje de población entre 25 y 64 años con titulación universitaria. El informe de la OCDE Education at a Glance de 2011 (Gráfico 1) nos muestra que en España había en 2009 un $30 \%$ de los ciudadanos entre 25 y 64 años con titulaciones universitarias. Ocupa el primer lugar en este indicador Canadá, donde la mitad de la población de este grupo de edad tiene una titulación universitaria. Le siguen Japón y EE. UU. con más de 4 de cada 10 personas. En relación a los países europeos destacan los del norte de Europa: Finlandia, Reino Unido, Noruega, Irlanda, Dinamarca. En el extremo inferior, están Italia y Portugal con un $15 \%$.
Gráfico 1.

Personas de 25 a 64 años con titulaciones universitarias en países de la OCDE (2009)

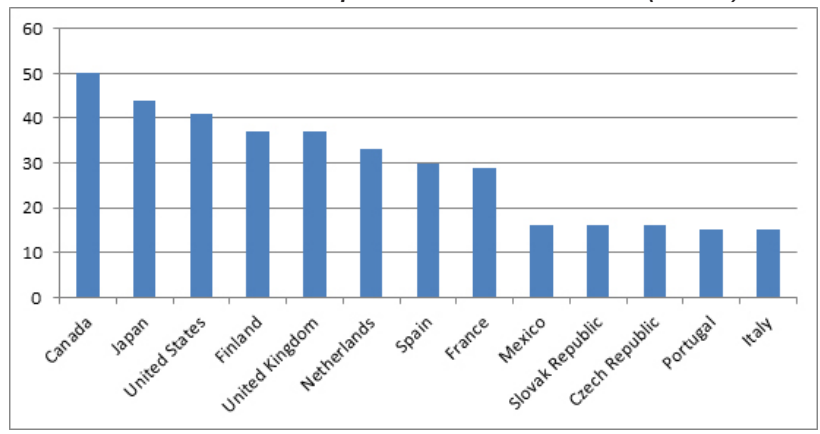

Fuente: Informe OCDE Education at Glance 2011. Eboración propia.

$\mathrm{Si}$ atendemos a las diferencias por Comunidades Autónomas (en adelante CC. AA.) según los datos de 2011 (Cuadro VIII) destaca la Comunidad de Madrid con un $39,4 \%$. También se sitúan por encima de la media País Vasco, Navarra y Cataluña. En el otro extremo de CC. AA. con menores porcentajes de universitarios, están Galicia, la Rioja, Canarias, Murcia y Castilla-La Mancha (menos del $24 \%$ de los ocupados).

Cuadro VIII.

Clasificación de CC. AA. por Titulaciones Universitarias (2011).

\begin{tabular}{|l|c|c|}
\hline Madrid & 39,4 & 100,0 \\
\hline País Vasco & 33,4 & 84,9 \\
\hline Navarra & 31,1 & 79,1 \\
\hline Cataluña & 30,9 & 78,6 \\
\hline Asturias & 29,6 & 75,3 \\
\hline Aragón & 28,5 & 72,4 \\
\hline Comunidad Valenciana & 28,1 & 71,4 \\
\hline Andalucía & 28,0 & 71,1 \\
\hline Castilla-León & 27,3 & 69,3 \\
\hline Cantabria & 26,9 & 68,5 \\
\hline Islas Baleares & 26,1 & 66,5 \\
\hline Extremadura & 26,0 & 66,0 \\
\hline Galicia & 25,6 & 65,0 \\
\hline La Rioja & 25,5 & 64,7 \\
\hline Canarias & 25,2 & 64,0 \\
\hline Murcia & 24,7 & 62,9 \\
\hline Castilla-La Mancha & 23,3 & 59,2 \\
\hline
\end{tabular}

Fuente: Censo 2011. Elaboración propia.

Si nos fijamos en las provincias (Cuadro IX), entre las 10 primeras provincias nos encontramos con Madrid, dos provincias del País Vasco, dos provincias de Castilla-León, dos provincias andaluzas, Navarra y otra de Aragón. Entre las diez provincias con menores universitarios tenemos a tres provincias gallegas, tres provincias de Castilla-La Mancha, tres provincias del Mediterráneo y una isleña. 
Cuadro IX.

Clasificación de provincias por Titulaciones Universitarias (2011)

\begin{tabular}{|l|c|l|c|}
\hline Madrid & 39,4 & Huesca & 25,6 \\
\hline Vizcaya & 35,0 & Santa Cruz de Tenerife & 25,6 \\
\hline Granada & 33,0 & Burgos & 25,5 \\
\hline Barcelona & 33,0 & La Rioja & 25,5 \\
\hline Valladolid & 32,4 & Albacete & 25,5 \\
\hline Guipúzcoa & 32,3 & León & 25,4 \\
\hline Sevilla & 31,5 & Lérida & 25,4 \\
\hline Navarra & 31,2 & Pontevedra & 25,0 \\
\hline Valencia & 31,2 & Las Palmas & 24,9 \\
\hline Salamanca & 31,2 & Alicante & 24,8 \\
\hline Álava & 30,7 & Murcia & 24,8 \\
\hline Zaragoza & 30,3 & Soria & 24,6 \\
\hline Asturias & 29,7 & Castellón & 24,5 \\
\hline Melilla & 28,5 & Ciudad Real & 24,3 \\
\hline La Coruña & 28,1 & Gerona & 24,2 \\
\hline Málaga & 27,8 & Huelva & 24,1 \\
\hline Ceuta & 27,3 & Palencia & 24,1 \\
\hline Cantabria & 27,0 & Almería & 23,7 \\
\hline Cádiz & 27,0 & Ávila & 23,5 \\
\hline Córdoba & 26,4 & Jaén & 23,4 \\
\hline Tarragona & 26,2 & Orense & 23,1 \\
\hline Islas Baleares & 26,2 & Zamora & 22,3 \\
\hline Badajoz & 26,1 & Cuenca & 21,6 \\
\hline Guadalajara & 25,9 & Lugo & 21,3 \\
\hline Cáceres & 25,9 & Toledo & 20,5 \\
\hline Segovia & 25,9 & Teruel & \\
\hline
\end{tabular}

Fuente: Censo de 2011. Elaboración propia.

El tercer y último indicador que utiliza Florida para medir el Talento es el Índice de Talento Científico, que mide el número de investigadores a jornada completa o equivalente por cada 1000 ocupados. En Europa, España, Italia, Grecia y Portugal ocupan los últimos lugares con menos de 10 investigadores por cada 1000 ocupados. En contraposición los países nórdicos, Finlandia y Suecia, tienen más de un $20^{8}$.

Dentro de España (Gráfico 2) se observan importantes diferencias por CC. AA. País Vasco ocupa el primer lugar con casi 13 ocupados. Le sigue muy cerca Navarra con más de 8. Por encima de la media española también se sitúan Navarra, País Vasco, Asturias, Castilla-León y Cataluña. Sin embargo, Castilla-León, Baleares, Canarias y Extremadura tienen menos de 4 investigadores por cada 1000 ocupados.

Por último, analizados los subíndices, es el momento de pasar a ver el índice de talento ${ }^{9}$. Como hemos ido viendo en este apartado hay enormes diferencias por CC. AA. (Cuadro X). En el año 2011, Madrid ocupa la primera posición en la clasificación en los tres indicadores. País Vasco y Navarra y conformarían el podio de talento con una puntuación por encima del $80 \%$. Por encima de la media también podemos situar a Asturias, Castilla-León, Cataluña y Aragón.
Gráfico 2.

Investigadores en I+D en por comunidades autónomas en \% (2011).

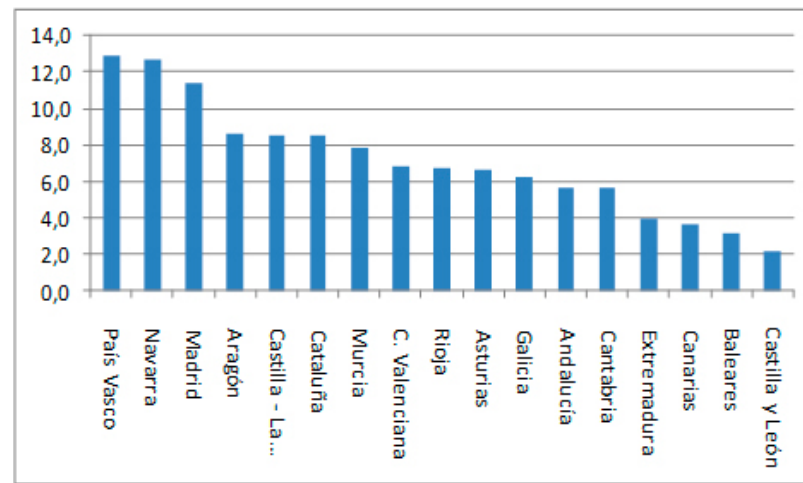

Fuente: Encuesta sobre actividades de I+D 2011. Elaboración propia.

Cuadro X.

Clasificación de talento por comunidades autónomas (2011).

\begin{tabular}{|l|c|c|c|c|}
\hline & Ocupados & Universitarios & Investigadores & $\begin{array}{c}\text { Clasificación } \\
\text { talento }\end{array}$ \\
\hline Madrid & 100,0 & 100,0 & 88,5 & 96,2 \\
\hline País Vasco & 83,1 & 84,9 & 100,0 & 89,3 \\
\hline Navarra & 76,3 & 79,1 & 98,1 & 84,5 \\
\hline Cataluña & 82,4 & 78,6 & 65,9 & 75,6 \\
\hline Aragón & 68,9 & 72,4 & 67,1 & 69,4 \\
\hline Castilla-León & 65,9 & 69,3 & 66,3 & 67,2 \\
\hline $\begin{array}{l}\text { Comunidad } \\
\text { Valenciana }\end{array}$ & 74,0 & 71,4 & 52,9 & 66,1 \\
\hline Asturias & 71,8 & 75,3 & 51,1 & 66,1 \\
\hline Murcia & 66,7 & 62,9 & 60,8 & 63,5 \\
\hline Andalucía & 73,2 & 71,1 & 44,0 & 62,8 \\
\hline Cantabria & 70,7 & 68,5 & 44,0 & 61,1 \\
\hline Galicia & 67,1 & 65,0 & 48,1 & 60,1 \\
\hline La Rioja & 63,2 & 64,7 & 52,2 & 60,0 \\
\hline Extremadura & 69,8 & 66,0 & 30,5 & 55,4 \\
\hline Baleares & 72,1 & 66,5 & 24,6 & 54,4 \\
\hline Canarias & 66,6 & 64,0 & 28,3 & 53,0 \\
\hline $\begin{array}{l}\text { Castilla-La } \\
\text { Mancha }\end{array}$ & 62,1 & 59,2 & 16,7 & 46,0 \\
\hline
\end{tabular}

Fuente: Censo de 2011. Elaboración propia.

Las comunidades autónomas que peores resultados obtienen en relación con el talento son CastillaLa Mancha, Canarias y Baleares, que se sitúan por debajo del $50 \%$ de Madrid. Por debajo del $60 \%$ de talento, se sitúan Murcia, Canarias, C. Valenciana, Extremadura y Galicia.

No existen datos desagregados a nivel provincial sobre el número de investigadores por cada 1000 ocupados, por lo tanto la clasificación de talento a nivel provincial se establece con solo dos indicadores: clases creativas y universitarios (Cuadro $\mathrm{XI}$ ). En la cúspide de la clasificación se sitúa la provincia de Madrid. Aparecen muy cerca Vizcaya y Barcelona con más de un $85 \%$ del talento de la provincia madrileña. En el intervalo del 85 y 75 se sitúan Granada, Sevilla, Guipúzcoa, Valladolid, Valencia, Navarra, Álava y Salamanca. Entre las provincias con datos más bajos en el índice de talento, por debajo del $60 \%$, observamos a Palencia, Orense, Zamora, Lugo, Cuenca, Toledo y Teruel. 
Cuadro XI.

Clasificación de talento por Provincias (2011).

\begin{tabular}{|c|c|c|c|c|c|c|c|}
\hline & $\begin{array}{l}0 \\
0 \\
0 \\
\pi \\
0 \\
\frac{0}{0} \\
0\end{array}$ & 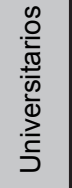 & $\begin{array}{l}\frac{.0}{0} \\
\frac{0}{0} \\
\frac{0}{4} \\
\frac{0}{0} \\
\frac{\pi}{U}\end{array}$ & & $\begin{array}{l}0 \\
0 \\
0 \\
\mathbb{0} \\
\frac{0}{3} \\
0 \\
0\end{array}$ & 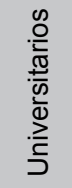 & 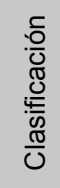 \\
\hline Madrid & 100 & 100 & 100,0 & Pontevedra & 69,0 & 63,3 & 66,2 \\
\hline Vizcaya & 86,7 & 88,9 & 87,8 & Lérida & 67,8 & 64,4 & 66,1 \\
\hline Barcelona & 87,6 & 83,7 & 85,6 & Alicante & 67,8 & 62,9 & 65,3 \\
\hline Granada & 83,9 & 83,8 & 83,8 & Guadalajara & 64,8 & 65,8 & 65,3 \\
\hline Sevilla & 84,3 & 79,9 & 82,1 & Murcia & 66,7 & 62,9 & 64,8 \\
\hline Guipúzcoa & 81,1 & 82,0 & 81,5 & Ciudad Real & 67,9 & 61,5 & 64,7 \\
\hline Valladolid & 78,2 & 82,3 & 80,2 & Huesca & 63,6 & 65,0 & 64,3 \\
\hline Valencia & 80,5 & 79,1 & 79,8 & Las Palmas & 65,1 & 63,2 & 64,1 \\
\hline Navarra & 76,3 & 79,1 & 77,7 & La Rioja & 63,2 & 64,7 & 64,0 \\
\hline Álava & 75,1 & 77,9 & 76,5 & Gerona & 65,9 & 61,3 & 63,6 \\
\hline Salamanca & 73,9 & 79,1 & 76,5 & Burgos & 61,0 & 64,8 & 62,9 \\
\hline Zaragoza & 72,2 & 76,8 & 74,5 & Castellón & 63,7 & 62,1 & 62,9 \\
\hline Asturias & 71,8 & 75,3 & 73,6 & Segovia & 59,9 & 65,7 & 62,8 \\
\hline La Coruña & 71,1 & 71,2 & 71,1 & León & 60,2 & 64,4 & 62,3 \\
\hline Málaga & 71,2 & 70,4 & 70,8 & Huelva & 63,5 & 61,2 & 62,3 \\
\hline Cantabria & 70,7 & 68,5 & 69,6 & Soria & 59,5 & 62,3 & 60,9 \\
\hline Baleares & 72,1 & 66,5 & 69,3 & Jaén & 62,2 & 59,3 & 60,8 \\
\hline Córdoba & 71,0 & 66,9 & 68,9 & Almería & 61,4 & 60,2 & 60,8 \\
\hline Cádiz & 68,6 & 68,4 & 68,5 & Ávila & 61,1 & 59,7 & 60,4 \\
\hline Cáceres & 70,9 & 65,7 & 68,3 & Palencia & 58,6 & 61,0 & 59,8 \\
\hline Tarragona & 69,0 & 66,5 & 67,7 & Orense & 59,2 & 58,5 & 58,9 \\
\hline Badajoz & 69,1 & 66,3 & 67,7 & Zamora & 57,6 & 56,5 & 57,0 \\
\hline Albacete & 70,5 & 64,7 & 67,6 & Lugo & 56,0 & 54,0 & 55,0 \\
\hline Badajoz & 69,1 & 66,3 & 67,7 & Cuenca & 54,6 & 54,7 & 54,6 \\
\hline Albacete & 70,5 & 64,7 & 67,6 & Toledo & 54,5 & 53,0 & 53,7 \\
\hline Tenerife & 68,4 & 64,9 & 66,6 & Teruel & 53,1 & 51,9 & 52,5 \\
\hline
\end{tabular}

Fuente: Censo 2011. Elaboración propia.

\section{TOLERANCIA}

La tolerancia es el indicador que más original hace la medición de la creatividad que realiza Richard Florida, a la vez que le da un carácter más específicamente sociológico. La razón de introducirlo es la suposición de que para que emerjan sujetos creativos es necesario un contexto social, pero entendido en términos de clima moral o ideoafectivo, que facilite la creatividad. El término "tolerancia" quizás no retrate del todo bien ese contexto pues tiene muchas connotaciones, además de graves y controvertidos problemas conceptuales (Villaverde Rico, Laursan 2011), que debieran resolverse, pero lo usaremos pues no creemos que sea este el punto en el que haya que distanciarse de Florida.
Merece la pena hacer algún comentario a la puntuación de España en el índice de tolerancia que elaboraron Florida y (2004: 26 y ss.) para los 14 países que iniciaron la aventura económica del euro y en el que incluyó también a Estados Unidos. Esta medición, distinta a la realizada en Estados Unidos, mide la racionalización y secularización de los valores, la autoexpresión y la actitud ante las minorías. Los dos primeros índices se inspiran en los trabajos de Inglehart sobre los valores postmaterialistas iniciados en los años 70 (Inglehart 1991), continuada en los 90 con la noción de "postmodernización" (Inglehart 2001) y culminada en la primera década del siglo XXI con datos relativos al "desarrollo humano", que se mide teniendo en cuenta el eje que va de los valores tradicionales a los secular-racionales (bastante relacionado con sus trabajos anteriores) y el que conecta los valores de supervivencia con los de autoexpresión (donde la influencia de Maslow es patente). (Inglehart y Welzel 2006) ${ }^{10}$.

En la clasificación de 15 España ocupó la posición 11, solo por encima de Grecia, Irlanda, Estados Unidos y Portugal, con 6,57 puntos sobre 15. La tabla final delataba claramente que los 7 primeros eran países protestantes del centro y norte de Europa y que, tras Francia y Bélgica, ocupaban la parte inferior 5 países católicos y Estados Unidos. En cuanto a la puntuación en los componentes de dicha tolerancia, España fue la primera en lo que se refiere a las actitudes hacia las minorías, Por lo que respecta a la racionalización y secularización de los valores, España fue la cuarta por abajo con un escuálido 0,84 , pero muy por encima de Estados Unidos que marco -4, así como de Irlanda y Grecia que estaban en -8. Finalmente, España fue la última en autoexpresión con un 3,78, por detrás de Portugal. En los dos últimos indicadores encabezó la clasificación Suecia, que también puntuó bien en el primero, pues fue segunda.

Aunque esta medición de la tolerancia es mucho mejor que la llevada a cabo para Estados Unidos, no nos ha sido posible aplicarla a España. La razón es que los datos de la World Values Survey solo tienen en cuenta los Estados nacionales y no unidades menores, tal como nosotros necesitamos. Por eso, al final, hemos decidido abordar el asunto de la tolerancia utilizando la vía que Florida aplicó en su investigación sobre la clase creativa llevada a cabo en Estados Unidos. Es decir, viendo la proporción de bohemios, extranjeros y homosexuales ${ }^{11}$.

En realidad, la presencia de extranjeros no es un indicador que nos hable de la tolerancia e incluso de la heterogeneidad de una sociedad ${ }^{12}$. Sin necesidad de ellos, un país como India, con más de 50 lenguas oficiales, un sistema religioso politeísta y una democracia estable es claramente heterogéneo y tolerante. Una heterogeneidad y tolerancia similares a la hindú parece que nos encontramos en la multicultural Londres, donde las escuelas públicas cuentan con un conjunto de alumnos que hablan hasta 300 lenguas 
diferentes. Lo mismo podríamos decir de Estados Unidos si tomamos en cuenta las mezclas de los diferentes grupos étnicos que componen el país y que contribuyen a hacerlo más heterogéneo, así como a aumentar su tolerancia. La cantidad de niños y niñas nacidos de familias raciales mixtas creció de 500.000 en 1970 a 2 millones en 1990 y a 6,8 millones (el 2,5 $\%$ de la población) según el censo del 2000. Con ese crecimiento también han aumentado las variantes de afro-americanos, asiático-americanos, hispanoamericanos, afro-asiático-hispano-americanos, etc. (Bergua 2009:226-227). En España aún andamos lejos del crisol cultural norteamericano, pero los matrimonios mixtos fueron en el 2005 el 10,76 \% del total (el $4,13 \%$ en 1996) y los nacimientos en los que al menos uno de los padres era extranjero ascendieron al $17,65 \%$ (el 4,54 \% en 1996). Algo lógico si se tiene en cuenta que en la década de los 80 , la entrada de inmigrantes se multiplicó por 3 y según el censo de 2001 el número de ellos en España era de 1,57 millones, cuatro veces más que en $1991^{13}$. Los datos del censo de 2011 aún son más sorprendentes ya que la población extranjera total asciende a 5,25 millones personas, lo cual es un ascenso más que considerable, en relación con las dos décadas anteriores.

Dice Florida, que la presencia de homosexuales también es un indicador del clima de tolerancia de una sociedad. No solo eso. Una de sus conclusiones más controvertidas y criticadas es que el porcentaje de población de gays y lesbianas de un territorio tiene relación directa con la creatividad e incluso con el crecimiento económico. Según el censo del 2011, de los 163.338 matrimonios registrados, apenas 3540 eran de homosexuales (2073 entre varones y 1467 entre mujeres).

Por lo que respecta a la población bohemia ${ }^{14}$, obviando las dificultades para comparar las cantidades de 1991 y 2001 disponibles ${ }^{15}$, se observa que entre las dos fechas el número se ha duplicado. Los incrementos mayores, por lo que respecta a las comunidades autónomas, los experimentó Madrid, donde faltó muy poco para duplicar sus bohemios con su aumento de 6 décimas. Le siguieron, en este orden, Cataluña (casi 5 décimas), Canarias (4) y País Vasco (3). Después hay 6 comunidades que aumentaron entre 1 y 2 décimas (Andalucía, Aragón, Asturias, Baleares, Comunidad Valenciana, Galicia y Melilla), 4 que se mantuvieron prácticamente igual (Cantabria, Castilla La Mancha, Murcia y Rioja), 2 que incluso disminuyeron una décima (Extremadura y Ceuta) y otra, Castilla-León, que retrocedió 2 . En definitiva se nos abre un espectro de cambio muy amplio que va desde el $66,6 \%$ de aumento de bohemios en Madrid al 28,5\% de descenso en Castilla-León. En cuanto a datos más actuales, el censo de 2011, Baleares se sitúa a la cabeza de la clasificación seguido de Canarias, Cataluña y Galicia.

Tras el repaso a los controvertidos componentes del indicador de tolerancia construido por Florida para medir la creatividad en Estados Unidos, los resultados que podemos ofrecer para España están en las clasificaciones por comunidades autónomas (Cuadro XII) y por provincias (Cuadro XIII).

Cuadro XII.

Clasificación de tolerancia por comunidades autónomas (2011).

\begin{tabular}{|l|c|c|c|c|c|}
\hline \multicolumn{1}{|c|}{ Comunidades Autónomas } & Clasificación & Homosexuales & Bohemios & Extranjeros & Sumatorio \\
\hline Baleares & 95,05 & 85,15 & 100,00 & 100,00 & 285,15 \\
\hline Canarias & 77,59 & 100,00 & 66,99 & 65,78 & 232,77 \\
\hline Cataluña & 73,03 & 92,34 & 52,41 & 74,35 & 219,10 \\
\hline Madrid & 65,68 & 80,83 & 43,27 & 72,93 & 197,03 \\
\hline Comunidad Valenciana & 64,82 & 71,74 & 47,88 & 74,84 & 194,47 \\
\hline La Rioja & 44,50 & 55,98 & 9,46 & 68,06 & 133,50 \\
\hline Murcia & 44,22 & 41,69 & 14,26 & 76,70 & 132,65 \\
\hline Castilla La Mancha & 43,78 & 30,81 & 49,85 & 50,68 & 131,34 \\
\hline Aragón & 43,72 & 30,24 & 40,19 & 60,72 & 131,15 \\
\hline Andalucía & 42,07 & 47,48 & 39,78 & 38,95 & 126,21 \\
\hline Navarra & 38,97 & 29,71 & 36,65 & 50,56 & 116,92 \\
\hline País Vasco & 35,90 & 42,26 & 33,27 & 32,18 & 107,71 \\
\hline Cantabria & 34,87 & 33,90 & 39,39 & 31,32 & 104,60 \\
\hline Galicia & 32,28 & 27,59 & 50,73 & 18,53 & 96,85 \\
\hline Castilla León & 28,00 & 23,17 & 29,00 & 31,84 & 84,01 \\
\hline Extremadura & 27,28 & 28,95 & 35,52 & 17,36 & 81,83 \\
\hline Asturias & 22,68 & 32,49 & 13,56 & 21,99 & 68,04 \\
\hline
\end{tabular}

Fuente: Censo de 2011. Elaboración propia. 
Cuadro XIII.

Clasificación de tolerancia por provincias (2011).

\begin{tabular}{|c|c|c|c|c|c|}
\hline Provincias & Clasificación & Homosexuales & Bohemios & Extranjeros & Sumatorio \\
\hline Islas Baleares & 91,64 & 76,58 & 100,00 & 98,35 & 274,93 \\
\hline Gerona & 79,55 & 54,92 & 84,91 & 98,83 & 238,66 \\
\hline Santa Cruz de Tenerife & 76,07 & 79,48 & 83,57 & 65,16 & 228,22 \\
\hline Alicante & 74,08 & 70,81 & 51,43 & 100,00 & 222,24 \\
\hline Las Palmas & 72,15 & 100,00 & 52,17 & 64,28 & 216,44 \\
\hline Barcelona & 71,29 & 94,61 & 52,43 & 66,84 & 213,88 \\
\hline Madrid & 62,56 & 72,70 & 43,27 & 71,72 & 187,69 \\
\hline Málaga & 61,77 & 59,55 & 50,59 & 75,18 & 185,32 \\
\hline Almería & 60,35 & 41,95 & 42,02 & 97,08 & 181,05 \\
\hline Guadalajara & 59,64 & 46,50 & 58,44 & 73,96 & 178,91 \\
\hline Tarragona & 59,36 & 56,64 & 36,19 & 85,25 & 178,07 \\
\hline Valencia & 55,76 & 64,44 & 50,25 & 52,60 & 167,29 \\
\hline Castellób & 51,99 & 46,16 & 27,86 & 81,95 & 155,97 \\
\hline Toledo & 51,36 & 36,52 & 62,73 & 54,83 & 154,08 \\
\hline Cuenca & 46,72 & 4,47 & 77,44 & 58,26 & 140,17 \\
\hline Zaragoza & 46,23 & 30,40 & 47,53 & 60,76 & 138,69 \\
\hline Segovia & 45,91 & 38,33 & 38,25 & 61,14 & 137,72 \\
\hline Lérida & 44,80 & 25,86 & 22,65 & 85,89 & 134,40 \\
\hline Murcia & 42,40 & 37,50 & 14,26 & 75,43 & 127,19 \\
\hline Álava & 42,34 & 53,57 & 29,95 & 43,48 & 127,01 \\
\hline La Rioja & 42,25 & 50,34 & 9,46 & 66,94 & 126,74 \\
\hline Navarra & 37,70 & 26,72 & 36,65 & 49,73 & 113,10 \\
\hline Sevilla & 37,52 & 44,49 & 49,02 & 19,05 & 112,55 \\
\hline Cádiz & 36,40 & 37,01 & 53,57 & 18,64 & 109,21 \\
\hline Huesca & 35,55 & 27,61 & 22,76 & 56,27 & 106,64 \\
\hline La Coruña & 35,16 & 29,65 & 59,88 & 15,95 & 105,48 \\
\hline Granada & 34,24 & 46,15 & 22,89 & 33,69 & 102,73 \\
\hline Orense & 33,62 & 5,65 & 72,53 & 22,67 & 100,86 \\
\hline Cantabria & 33,56 & 30,49 & 39,39 & 30,80 & 100,68 \\
\hline Vizcaya & 33,13 & 35,13 & 34,45 & 29,82 & 99,40 \\
\hline Huelva & 32,80 & 39,14 & 19,94 & 39,33 & 98,41 \\
\hline Guipúzcoa & 32,71 & 35,90 & 32,96 & 29,28 & 98,14 \\
\hline Salamanca & 32,56 & 23,80 & 51,19 & 22,68 & 97,67 \\
\hline Pontevedra & 32,07 & 27,95 & 49,28 & 18,98 & 96,21 \\
\hline Burgos & 30,63 & 19,61 & 29,88 & 42,40 & 91,89 \\
\hline Ciudad Real & 29,02 & 13,68 & 35,56 & 37,80 & 87,05 \\
\hline Badajoz & 28,83 & 29,17 & 40,70 & 16,61 & 86,49 \\
\hline León & 27,29 & 20,52 & 37,04 & 24,32 & 81,88 \\
\hline Ávila & 26,74 & 21,25 & 20,68 & 38,30 & 80,23 \\
\hline Albacete & 26,72 & 22,68 & 20,56 & 36,93 & 80,17 \\
\hline Palencia & 24,18 & 20,63 & 31,26 & 20,66 & 72,55 \\
\hline Soria & 23,35 & 9,95 & 10,58 & 49,52 & 70,04 \\
\hline Teruel & 21,89 & 0,00 & 7,63 & 58,04 & 65,67 \\
\hline Valladolid & 21,48 & 20,73 & 15,41 & 28,31 & 64,45 \\
\hline Cáceres & 21,48 & 19,50 & 27,10 & 17,84 & 64,44 \\
\hline Asturias & 21,47 & 29,22 & 13,56 & 21,62 & 64,40 \\
\hline Córdoba & 21,05 & 33,03 & 15,71 & 14,40 & 63,14 \\
\hline Jaén & 19,55 & 18,23 & 24,09 & 16,32 & 58,64 \\
\hline Zamora & 15,09 & 5,37 & 19,12 & 20,78 & 45,28 \\
\hline Lugo & 12,53 & 12,10 & 6,13 & 19,37 & 37,60 \\
\hline
\end{tabular}

Fuente: Censo de 2011. Elaboración propia. 
En el primero tenemos a Islas Baleares $(95,05)$, que asciende dos puestos frente a la clasificación del 2001, en el cual se situaba en primer lugar Cataluña $(78,9)$, comunidad que le cambia el puesto y baja a un tercer lugar en este 2011 con 73,03 puntos. Este primer puesto debe su alta puntuación a haberse situado en primer lugar en los subíndices de bohemios y extranjeros. En segundo lugar y con un notable alto, gracias a su alta puntuación en población homosexual, se encuentra Canarias $(77,59)$, comunidad que una década antes se situaba en una cuarta posición con resultados inferiores $(55,4)$. En cuarto y quinto lugar, con alrededor de 65 puntos, aparecen Comunidad de Madrid y Comunidad Valenciana, las cuales obtienen notables en extranjeros y homosexuales, pero suspenden en población bohemia. A partir de aquí siguen rondando el suspenso alto, La Rioja $(44,50)$, Murcia $(44,22)$, Castilla-La Mancha $(43,78)$, Aragón $(43,72)$ y Andalucía $(42,07)$. Ya en zona de suspensos más altos, alrededor de 30 puntos, nos encontramos en orden descendente a Navarra, País Vasco, Cantabria y Galicia. No obstante, en algún subíndice de estas comunidades aparecen aprobados, como en el caso de los bohemios en Galicia. Las últimas posiciones de la clasificación las ocupan CastillaLeón $(28)$, Extremadura $(27,28)$ y Asturias $(22,68)$. Sorprendentemente en el censo de 2001 también se encontraban en las últimas posiciones, pero con menor puntuación, Castilla-León y Extremadura, con 17,9 y 16,4 puntos respectivamente.

En general comprobamos que en la puntuación general de las comunidades que están en posiciones superiores, pesa bastante la parte extranjera de la tolerancia, sobre todo en Murcia, donde su peso relativo es muy superior a ninguna otra Comunidad, al igual que ocurría diez años atrás. Otro aspecto importante es que el peso de la homosexualidad tiende a disminuir casi progresivamente desde la primera a la última posición de la clasificación. En relación a este componente sobresale Canarias que, sin duda, debe su primera posición a la gran importancia que en él tienen los matrimonios homosexuales (en 2001 era Cataluña la que se beneficiaba del peso de este indicador). Finalmente, por lo que respecta al componente bohemio, comprobamos que el peso es bastante similar y débil en casi todas las Comunidades (entre 30-50 puntos). Entre los volúmenes superiores sobresalen Baleares, Canarias, Cataluña y Cantabria. Curiosamente Madrid pierde importancia en población bohemia frente a 2001, año en la que era la comunidad más bohemia, frente a Murcia, que tenía menos bohemios que el resto de las comunidades más tolerantes. Sin embargo en 2011 es La Rioja la comunidad con menor volumen en población bohemia.

Por provincias (Cuadro XIII) destaca Baleares $(91,64)$, a la que le sirven las máximas puntuaciones que obtiene en bohemios y extranjeros y, en un notable alto, en extranjeros. Le sigue Gerona, que pun- túa más abajo por su baja puntuación en el indicador de homosexuales, frente a los magníficos resultados que obtiene en los otros dos indicadores. En las siguientes cuatro provincias, que son Santa Cruz de Tenerife $(76,07)$, Alicante $(74,08)$, Las Palmas $(72,15)$ y Barcelona $(71,29)$ resaltan las altas puntuaciones presentes en alguno de los tres subíndices de las mismas, como es el caso de los 100 puntos que obtiene Alicante en extranjeros y Las Palmas en homosexuales, o los 94,61 puntos de Barcelona también en este último. A pesar de estos datos, no se han situado en puestos superiores por tener en común puntuaciones más débiles en población bohemia. Por ello, podríamos considerar que el indicador de bohemios es determinante en los resultados finales del indicador de tolerancia, sobre todo en las provincias que alcanzan mejores medias finales.

\section{Creatividad}

A continuación (Cuadro XIV), mostramos en orden descendente, los resultados provinciales de creatividad y de sus tres componentes. Como se ve, hemos dejado fuera de la clasificación a Ceuta y Melilla. La razón es que, al ser simultáneamente ciudades y provincias, cambian la percepción de los análisis finales, pues en cada provincia se incluye también la población rural que, en general, parece hacer descender los 9 subíndices considerados, así que Ceuta y Melilla como son ciudades sin apenas entorno rural compiten con ventaja y obtienen indicadores superiores. También hemos incluido datos de la creatividad en 2001 obtenidos con otra investigación (Bergua 1015). Comprobamos que las puntuaciones en general bajan considerablemente (30 puntos lo hace la líder, Madrid) y que encabezan la clasificación del 2011 dos territorios que también puntuaban alto en el 2001, Madrid y Barcelona, por lo que la crisis no les ha afectado mucho. Sin embargo, irrumpen en la parte alta con aumentos espectaculares Gerona, Tenerife, Las Palmas, Málaga y Almería. En general, quienes suben en la clasificación y en puntuación son las islas, cuatro provincias mediterráneas (las mencionadas y Cádiz), otras cuatro de Castilla-La Mancha (Ciudad Real, Albacete, Cuenca y Toledo), solo una de Castilla León (Ávila) y otra de la Andalucía interior (Jaén). Si miramos la parte baja de la clasificación, no cambian mucho las posiciones (Ávila, Palencia, Jaén Teruel y Lugo tienen, en ambas fechas, puntuaciones mínimas). Sin embargo, si observamos a las que pierden posición y puntos comprobamos que Navarra, País Vasco, Aragón, tres provincias gallegas, otras tantas catalanas, Cantabria y gran parte de Castilla León salen muy mal paradas. Finalmente, no hay provincias que pierdan posición en la clasificación y ganen puntuación, pero sí quienes ganan clasificación perdiendo puntaje (Valencia, La Coruña, Sevilla y Segovia -que apenas pierde 0,07 puntos-, pero se podría añadir Málaga que apenas gana 0,19). En definitiva, se observa 
que el declive general de la creatividad en España ha permitido mejorar a las islas y el Mediterráneo en general y ha perjudicado al norte e interior. Todo ello como consecuencia de que el índice de tolerancia se ha comportado ahí mejor, permitiendo, a quienes les ha ido bien, medrar; y bajar a quienes no les ha ido tan bien. Madrid y Barcelona han sorteado mejor que otros territorios el descenso general porque no andaban mal en tolerancia.

Por lo que respecta a las Comunidades Autónomas, (Cuadro XV) de nuevo se observa, pero de un modo más nítido, que el aumento de puntos y de posiciones, así como el descenso en ambos apartados, tienden a congregar todos los movimientos, salvo Valencia (que gana posición y pierde puntos). La Comunidad Autónoma que más mejora es Castilla-La Mancha, acompañada por las islas (Baleares y Canarias), Andalucía y Cantabria. Las que más pierden en ambos apartados son claramente la parte norte de la península (Cataluña, País Vasco, Navarra, Aragón, La Rioja y Castilla-León), la mayoría de ellas (salvo Cataluña) con poca puntuación en Tolerancia.

\section{Correlaciones}

En la investigación que inspira este artículo comprobamos que en la medición de la creatividad europea casi todos los componentes tenían un efecto positivo sobre el indicador general. Sin embargo, la mayor sorpresa nos la dio la correlación negativa $(-0,575)$ que observamos en Europa entre las actitudes hacia los extranjeros y la alta tecnología. Aunque en menor medida, también observamos que presentaba problemas otro de los componentes de la tolerancia, los valores, que solo obtenía tres coeficientes positivos y significativos. Pero es que tampoco la autoexpresión, el tercer componente de la creatividad en Europa, con cinco coeficientes positivos y significativos, salía bien parada. En definitiva, las tres variables que componían el subíndice de Tolerancia eran las de menor correlación con el resto de variables. De ahí que su correlación con el Índice de Creatividad fuera el de menor cuantía, 0,673, frente al 0,882 de la Tecnología y al 0,808 del Talento. Por lo tanto, la cuestión de la tolerancia, precisamente la que confiere mayor originalidad y da un carácter más sociológico a las tesis de Florida, es la que menos influye en el indicador final de creatividad.

En el índice de Tolerancia elaborado para el caso de las comunidades autónomas y provincias españolas, construido como el de Estados Unidos, observamos que obtenía mejores resultados (Cuadro $\mathrm{XVI}$ ). Sin embargo, también comprobamos que la tolerancia tiene una menor correlación significativa con la creatividad $(0,678)$ que el talento $(0,817)$ y la tecnología $(0,942)$. Igualmente se observa que los bohemios son los principales responsables de los buenos resultados, ya que sus coeficientes son todos positivos y significativos, tanto en las CC. AA.
Cuadro XIV.

Clasificación de creatividad provincial (2001).

\begin{tabular}{|c|c|c|c|c|}
\hline & $\begin{array}{c}\text { Clasificación } \\
2011\end{array}$ & $\begin{array}{c}\text { Clasificación } \\
2001\end{array}$ & $\begin{array}{l}\text { Diferencia } \\
\text { Posición }\end{array}$ & $\begin{array}{c}\text { Diferencia } \\
\text { Puntos }\end{array}$ \\
\hline Madrid & $54,18(1)$ & 84,0 (1) & $=$ & $-30,00$ \\
\hline Islas Baleares & $53,64(2)$ & $44,5(17)$ & +15 & $+9,14$ \\
\hline Barcelona & $52,29(3)$ & $69,7(2)$ & -1 & $-16,80$ \\
\hline Gerona & $47,71(4)$ & $44,3(19)$ & +15 & $+3,14$ \\
\hline Tenerife & $47,55(5)$ & $39,0(25)$ & +20 & $+8,41$ \\
\hline Alicante & $46,46(6)$ & $57,0(6)$ & $=$ & $-10,54$ \\
\hline Las Palmas & $45,41(7)$ & $38,9(26)$ & +19 & $+6,52$ \\
\hline Valencia & $45,18(8)$ & $50,7(12)$ & +4 & $-5,52$ \\
\hline Málaga & $44,19(9)$ & $43,2(20)$ & +11 & $+0,19$ \\
\hline Tarragona & $42,35(10)$ & $52,7(10)$ & $=$ & $-10,35$ \\
\hline Zaragoza & 42,24 (11) & $63,5(3)$ & -8 & $-21,26$ \\
\hline Guadalajara & $41,64(12)$ & $40,0(23)$ & +9 & $+1,64$ \\
\hline Almería & $40,38(13)$ & $35,9(30)$ & +17 & $+4,48$ \\
\hline Vizcaya & $40,27(14)$ & $53,6(9)$ & -5 & $-13,33$ \\
\hline Lérida & 39,96 (15) & $45,6(14)$ & -1 & $-5,64$ \\
\hline Sevilla & $39,87(16)$ & $41,2(22)$ & +6 & $-1,33$ \\
\hline Álava & $39,61(17)$ & $58,1(5)$ & -12 & $-18,49$ \\
\hline Granada & $39,28(18)$ & $51,0(11)$ & -7 & $-11,72$ \\
\hline Castellón & $38,29(19)$ & $56,5(7)$ & -12 & $-18,21$ \\
\hline Navarra & $38,13(20)$ & $63,2(4)$ & -16 & $-25,07$ \\
\hline Guipúzcoa & 38,07 (21) & $54,6(8)$ & -13 & $-16,53$ \\
\hline Salamanca & $36,35(22)$ & $39,4(24)$ & +2 & $-3,05$ \\
\hline Segovia & $36,23(23)$ & $36,3(29)$ & +6 & $-0,07$ \\
\hline Murcia & $35,73(24)$ & $43,1(21)$ & -3 & $-7,37$ \\
\hline Coruña & $35,46(25)$ & $38,6(27)$ & +2 & $-3,17$ \\
\hline La Rioja & $35,41(26)$ & $48,2(13)$ & -13 & $-12,79$ \\
\hline Toledo & $35,11(27)$ & $28,3(46)$ & +19 & $+6,81$ \\
\hline Cádiz & $34,96(28)$ & $31,2(38)$ & +10 & $+3,76$ \\
\hline Cantabria & $34,38(29)$ & $37,6(28)$ & -1 & $-3,22$ \\
\hline Valladolid & $33,89(30)$ & $45,2(16)$ & -14 & $-11,22$ \\
\hline Cuenca & 33,77 (31) & $30,0(42)$ & +11 & $+3,77$ \\
\hline Huesca & $33,28(32)$ & $35,6(32)$ & $=$ & $-2,32$ \\
\hline Pontevedra & 32,75 (33) & $35,8(31)$ & -2 & $-3,05$ \\
\hline Badajoz & $32,17(34)$ & $29,9(43)$ & +11 & $+2,27$ \\
\hline Huelva & $31,70(35)$ & $30,3(41)$ & +6 & $+1,40$ \\
\hline Asturias & $31,69(36)$ & $45,4(15)$ & -21 & $-13,71$ \\
\hline Albacete & $31,44(37)$ & $30,7(49)$ & +12 & $+0,74$ \\
\hline Ci. Real & $31,24(38)$ & $29,9(44)$ & +16 & $+1,34$ \\
\hline Burgos & $31,17(39)$ & $44,4(18)$ & -21 & $-13,23$ \\
\hline Orense & $30,84(40)$ & $33,4(34)$ & -6 & $-2,56$ \\
\hline Córdoba & $29,98(41)$ & $35,3(33)$ & -8 & $-5,32$ \\
\hline Cáceres & $29,92(42)$ & $31,0(39)$ & -3 & $-1,08$ \\
\hline León & $29,86(43)$ & $32,9(35)$ & -8 & $-3,04$ \\
\hline Ávila & $29,04(44)$ & $27,6(49)$ & +5 & $+1,44$ \\
\hline Soria & $28,08(45)$ & $32,9(36)$ & -9 & $-4,82$ \\
\hline Palencia & $27,99(46)$ & $31,4(37)$ & -9 & $-3,41$ \\
\hline Jaén & $26,78(47)$ & $26,3(50)$ & +3 & $+0,48$ \\
\hline Teruel & $24,79(48)$ & $29,8(45)$ & -3 & $-5,01$ \\
\hline Zamora & $24,03(49)$ & $28,2(47)$ & -2 & $-4,17$ \\
\hline Lugo & $22,51(50)$ & $27,9(48)$ & -2 & $-5,39$ \\
\hline
\end{tabular}

Fuente: INE; Oficina Española de Patentes y Marcas; Bergua (2015). Elaboración propia. 
Cuadro XV.

Índice de creatividad por CC. AA. (2001).

\begin{tabular}{|l|c|c|c|c|}
\hline & $\begin{array}{c}\text { Clasificación } \\
\mathbf{2 0 1 1}\end{array}$ & $\begin{array}{c}\text { Clasificación } \\
\mathbf{2 0 0 1}\end{array}$ & $\begin{array}{c}\text { Diferencia } \\
\text { posición }\end{array}$ & $\begin{array}{c}\text { Diferencia } \\
\text { puntos }\end{array}$ \\
\hline Madrid & $53,96(1)$ & $88,1(1)$ & $=$ & $-34,14(1)$ \\
\hline Islas Baleares & $49,81(2)$ & $43,0(8)$ & +6 & $+6,81(16)$ \\
\hline Cataluña & $49,54(3)$ & $72,2(2)$ & -1 & $-22,66(4)$ \\
\hline Valencia & $43,61(4)$ & $44,8(6)$ & +2 & $-1,19(11)$ \\
\hline Canarias & $43,53(5)$ & $42,3(9)$ & +4 & $+1,23(12)$ \\
\hline País Vasco & $41,70(6)$ & $64,9(4)$ & -2 & $-23,20(3)$ \\
\hline Navarra & $41,15(7)$ & $67,6(3)$ & -4 & $-26,45(2)$ \\
\hline Aragón & $37,70(8)$ & $47,8(5)$ & -3 & $-10,10(6)$ \\
\hline Castilla La \\
Mancha & $37,56(9)$ & $29,5(17)$ & +8 & $+8,06(17)$ \\
\hline Murcia & $35,90(10)$ & $39,2(12)$ & +3 & $+3,30(15)$ \\
\hline La Rioja & $34,83(11)$ & $44,1(7)$ & -3 & $-9,27(7)$ \\
\hline Andalucía & $34,27(12)$ & $36,4(13)$ & +2 & $+2,13(13)$ \\
\hline Cantabria & $31,99(13)$ & $35,8(15)$ & +3 & $+3,81(14)$ \\
\hline Castilla León & $31,73(14)$ & $40,6(11)$ & -2 & $-8,87(8)$ \\
\hline Galicia & $30,79(15)$ & $36,0(14)$ & $=$ & $-5,21(9)$ \\
\hline Asturias & $29,56(16)$ & $40,9(10)$ & -5 & $-11,34(5)$ \\
\hline Extremadura & $27,56(17)$ & $29,8(16)$ & $=$ & $-2,24(10)$ \\
\hline
\end{tabular}

Fuente: INE; Oficina Española de Patentes y Marcas; Bergua (2015). Elaboración propia.

\section{Cuadro XVI.}

Coeficientes de correlación.

\begin{tabular}{|l|c|c|c|}
\hline & Tecnología & Talento & Tolerancia \\
\hline Creatividad & 0,9420 & 0,8164 & 0,6779 \\
\hline Tecnología & & 0,8591 & 0,4243 \\
\hline Talento & & & 0,1957 \\
\hline
\end{tabular}

como en las provincias. Esto parece indicar que los problemas están en los porcentajes de población extranjera y homosexual, lo cual cuestionaría otra de las conclusiones de Florida, las relaciones positivas y significativas del índice gay-bohemio con el ingreso regional, los salarios, la tecnología y el capital humano (Florida y Mellander 2009).

\section{Conclusiones y discusión}

La clase creativa de España está entre el 23,9 \% (2001) y el $21,02 \%$ (2011) de la población, quedando algo lejos de Estados Unidos (30\%) y de los países del centro y norte de Europa (28-29\%), aunque por encima de Italia y Portugal (15\%). El territorio español más homologable a la pauta media europea es Madrid, con un $25 \%$ de clase creativa.

Madrid, País Vasco y Navarra muestran un claro dominio en Tecnología y Talento. Les acompañan Cataluña y Aragón. Algo más lejos, pero por encima del resto, están Asturias, Cantabria y Castilla-León.
De modo que hay un claro dominio del Norte y Este de España. Por provincias solo alteran este paisaje Castellón en Tecnología y Granada en Talento.

En relación a la Tolerancia, en el 2001 aparecían arriba comunidades que puntuaban bajo en los otros dos índices (Baleares, Canarias, Comunidad Valenciana y Murcia), bajaban otras que en talento y tecnología iban bien (Aragón y País Vasco) y se mantenían Madrid Cataluña y Navarra. Por provincias destacaba Alicante por encima del resto. En el 2011 los cambios en las puntuaciones y la clasificación se han producido por la pérdida de peso en tecnología y talento de los territorios punteros y el incremento de peso de la tolerancia, principalmente por lo que respecta a los extranjeros, en Comunidades que andaban bastante abajo (Baleares y Canarias).

Por otro lado, comprobamos que el subíndice de bohemios es el que mejor se relaciona con la Tecnología y el Talento, mientras que la proporción de homosexuales y extranjeros crea una jerarquización distinta. La excepción son Madrid y Barcelona.

Vemos también que la tolerancia combina mal con los otros índices y con la creatividad en general. Esto lleva a cuestionar la originalidad del indicador, pues se diferencia de otros precisamente en que introduce los valores y la heterogeneidad social. El País Vasco, con una magnífica puntuación en tecnología y talento pero baja en diversidad es un magnífico ejemplo de esto.

Quizás haya que mirar de otro modo esa influencia de la tolerancia, diversidad o heterogeneidad social y encontrarla en otros sitios. Por ejemplo, en lugar de medirla cuantitativamente sugerimos indagarla de un modo cualitativo. En concreto creemos que es necesario hacer historias de vida a partir de personajes que representen las 18 ocupaciones que en España se incluyen en la clase creativa según la considera Florida. En dichas historias se trataría de medir la riqueza de experiencias en cada biografía ${ }^{16}$, averiguar si las vidas de los informantes difieren entre sí y comprobar si transcurren sobre una trayectoria social también plural. En definitiva, se trataría de aplicar una sociología que preste atención a "la pluralidad de las lógicas efectivas de acción" y a "la pluralidad de las formas de relación de la acción" (Lahire 2004:227). Esto es importante porque solo desde esa pluralidad pueden generarse mecanismos productores de creatividad, como la bisociación (Koestler 1975: 45), consistente en la interactuación de dos matrices independientes de percepción o razonamiento, o el "pensamiento divergente" (Gardner 2010: 44) o "lateral" (De Bono 2006), caracterizados por seguir caminos alternativos o desacostumbrados para resolver problemas.

Por otro lado, además de a la heterogeneidad y diversidad social así entendidas, debiera prestarse atención a lo imaginario, imprescindible para que 
haya creatividad según Castoriadis (1989) y caracterizado por desafiar las lógicas que operan con identidades, distinciones, exclusiones o conjuntos, en la base del pensamiento científico y de la propia sociedad en tanto que institución. Es probable que lo imaginario así entendido esté detrás de esa placentera "experiencia flujo" que aparece en los momentos de creación y que se caracteriza por que el espacio y el tiempo dejan de percibirse y se siente una armonía interna en la que todo el ser está involucrado (Csíkszentmihályi 1996). Para descubrir y analizar todo esto serían necesarias medidas y modos de pensar más arriesgados y audaces.

\section{NOTAS}

1. En la actualidad no es posible entender las clases creativas sin hacer referencia a otras dos expresiones, la "economía creativa" y las "industrias creativas". En la mayoría de los países existen hoy alrededor de 14 sectores de economía creativa (la publicidad, la arquitectura, las artes, la artesanía, el diseño, la moda, la edición, la cinematografía y el vídeo, la televisión y la radio, los programas informáticos interactivos de ocio, la música, las artes de interpretación y ejecución, la fotografía, los servicios informáticos, etc.) que tienen un elevado componente artístico. Pero, en realidad, la creatividad, dice Hawkins (2005), existe prácticamente en todos los ámbitos de la vida colectiva. La otra expresión, "las industrias creativas", ha sido impulsada por la Unesco (2005) con la intención de sustituir un concepto ya caduco por estrecho, como es el de las "industrias culturales", y que hace referencia a aquellas industrias que combinan la creación, la producción y la comercialización de contenidos creativos que son intangibles y de naturaleza cultural. Supone entonces un conjunto más amplio de actividades, pues incluye a las industrias culturales (sectores editorial, multimedia, audiovisual, fonográfico, producciones cinematográficas, artesanía y diseño) más toda la producción artística o cultural, ya sean espectáculos o bienes producidos individualmente. En los países más desarrollados todos estos sectores crecen entre un $5 \%$ y un $20 \%$ anual.

2. Hay pues dos modos distintos de afrontar la existencia individual y colectiva. A un lado el sedentario, habitante de espacios que están estriados a base de prescripciones y proscripciones; al otro el nómada, que se mueve por espacios lisos en los que cualquier trayectoria puede ser trazada. No solo eso, cuando el sedentario visita los espacios lisos tiende inercialmente a estriarlos y cuando el nómada desembarca en los estriados su particular habitus le lleva a alisarlos (Deleuze y Guattari 1986: 483-506). El sedentarismo y el estriaje, del mismo modo que la sociedad del riesgo, son una resistencia. No solo al nomadismo y al alisamiento, también a la creatividad.

3. Jünger (1993: 52-61) opina igual. En su opinión, el burgués, llevado por un antiquísimo "afán de seguridad" se ha dedicado a "obturar el espacio vital" para impedir que "lo elemental" irrumpa. "La situación ideal de seguridad que el progreso aspira a alcanzar consiste en que el mundo sea dominado por la razón, la cual deberá no solo aminorar las fuentes de lo peligroso sino también, en última instancia, secarlas". No obstante, el plan ha tenido efectos perversos pues "en igual proporción que el orden sabe expulsar de sí el peligro, en esta mis-

\section{Agradecimientos}

El presente artículo es resultado de la investigación "Clases creativas en España: composición, formación y creatividad", a realizar entre 2011 y 2013, financiada por el Ministerio de Economía y Ciencia (ref. CSO2010-17139).

Agradecemos las aportaciones de los evaluadores anónimos de esta revista. Este trabajo recibió uno de los cinco galardones a los mejores artículos presentados en el VI Congreso Andaluz de Sociología, que tuvo lugar en la Universidad de Cádiz del 29 de noviembre al 1 de diciembre de 2012. ma medida tórnase este más amenazador y mortal". La forma que adquiere el retorno de esas fuerzas es la "anarquía". Añade Jünger que ese retorno de lo expulsado ha de dar lugar, una vez que se tome conciencia de su procedencia, a unos órdenes nuevos en los que esté incluido "lo extraordinario".

4. E incluso frívolos según algunos críticos de Florida, como Peck (2005). "Mezcla elitismo cosmopolita y universalismo pop, hedonismo y responsabilidad, radicalismo cultural y conservadurismo económico, libertarismo social y realismo de negocios". Todo ello tiene ecos de "las guías de estilo de vida, manuales empresariales y sociología pop de los manuales de nueva era económica".

5. El concepto "clase" incide en las relaciones de dependencia entre unos grupos sociales y otros, mientras que el "estrato" analiza la clase atendiendo a la relación de orden. Los dos enfoques de clase más reconocidos son los de Goldthorpe (2010), de inspiración weberiana y el de Wrigth (1983), neomarxista. Feito (1995: 139-143) disecciona las diferencias principales entre estos enfoques que, en resumen, puntúan en dos tipos distintos de relaciones, las de dependencia y las de orden. Siguiendo a Tezanos (2001: 95), la clase social "es un grupo social relativamente homogéneo en sus condiciones laborales y en sus intereses económicos, que ocupa una posición determinada de poder, de influencia y de oportunidades en la estructura social, en un momento histórico determinado de la evolución de los sistemas productivos, entendidos como sistemas sociales generales". Por tanto, no podemos entender la clase creativa como clase social puesto que no es un grupo homogéneo ni en sus condiciones laborales ni en sus intereses económicos. En el enfoque de Florida las clases sociales se comprenden simplemente como agrupamientos, principalmente ocupacionales y las estructuras de clase son fundamentalmente estructuras de desigualdad social. Son precisamente estas desigualdades las que convierten las diferencias de clase en resultados entre una serie de oportunidades y elecciones vitales en nuestras biografías (Goldthorpe 2010: 411).

6. El problema que observa el propio Florida y en el que inciden otras investigaciones (Donnegan y Low 2008) es la relación directamente proporcional entre tamaño de la clase creativa y desigualdad social. De todas formas, en la relación al género, Negrey y Rauchs 2009) han observado en Estados Unidos que la brecha varones/ mujeres ocupados es menor en la clase creativa que en la trabajadora y de servicios, independientemente de que la región considerada sea más o menos creativa. 
7. En su aproximación al caso español, Casares (2010: 72) utiliza como fuente de información básica del talento los datos del Censo de Población de 2001. En nuestro caso, también analizamos la clase creativa atendiendo a los criterios de la Clasificación Nacional de Ocupaciones 1994 (CNO-94). Los criterios de clasificación utilizados son dos: tipo de trabajo realizado y cualificación. Para esta clasificación, se entiende por cualificación "la capacidad para desempeñar las tareas inherentes a un empleo determinado". Para ello, tienen en cuenta dos puntos vista: el nivel y la especialización de dicha cualificación. No obstante, creemos que no debemos excluir las ocupaciones de la categoría 29 "Otras profesiones asociadas a una titulación de 1 er ciclo universitario" ya que encontramos muchas similitudes con otras ocupaciones que requieren diplomaturas universitarias que han sido incluidas en otros grupos ocupacionales.

8. Para ampliar datos véase Florida y Tinagli (2004).

9. En el estudio de Florida y Tinagli (2004), España ocupa el noveno lugar sobre un total de 15 países. Sin embargo, es el cuarto país en el indicador de capital humano (titulados universitarios). La peor posición la otorga el indicador de talento científico donde ocupa la undécima posición sobre 15 países.

10. Florida e Inglehart no parecen estar al tanto del auge de la noción "postsecular" (Beriáin y Sánchez de la Yncera 2012: 31-92), que hace referencia al fracaso institucional de la experiencia europea de la modernidad, que pretendió diferenciar las esferas seculares (Estado, economía, política) de las religiosas, asegurar que lo religioso estaba en declive e interpretar lo religioso como algo privado (Casanova 2012: 93-124).

11. Este indicador incluso ha sido utilizado en China para confirmar una correlación fuerte con el tamaño de la clase creativa y el volumen de capital humano (Qian 2010). De todas formas, este es un asunto polémico. Para algunos geógrafos, por ejemplo, la diversidad social no siempre mejora las ciudades. Es lo que parece ocurrir en Hamburgo (Pohl 2008).

12. Quizás sea por eso que en un reciente trabajo, Florida y Mellander (2009) solo usan un índice combinado de bohemios y gais para probar su influencia en el incremento del valor de la vivienda.

13. El $44 \%$ eran europeos y entre ellos dominaban los de la Europa del euro (33\%). Una proporción similar, el $45 \%$, tenían los americanos, siendo los del centro-sur los más abundantes (43\%). Los africanos suponían el $22 \%$ y los asiáticos apenas el $5 \%$. En la actualidad distintos informes coinciden en que el $8 \%$ de la pobla-

\section{ReferenCias Bibliográficas}

Badiou, A. 1990. ¿Es posible la política?. Buenos Aires: Nueva visión.

Badiou, A. 1999. Ser y acontecimiento. Buenos Aires: Manantial.

Beck, U. 2002. La sociedad del riesgo. Hacia una nueva modernidad. Barcelona: Paidós.

Bergua, J. A. 2009. Sociología de la política. Zaragoza: Mira. PMCid:PMC2738716

Bergua, J.A. -dir-. 2015. Creatividad. Números e imaginarios. Madrid: CIS (pendiente de publicación).

Beriáin, J. e I. Sánchez de la Yncera. 2012. "Tiempos de postsecularidad: desafíos de pluralismo para la teoría". Pp. 3192 en Dialécticas de la postsecularidad. Pluralismo y corrientes de secularización, editado por I. Sánchez de la Yncera y M. Rodríguez Fouz. Barcelona: Anthropos. ción española es extranjera. De ellos 1,6 millones (el $43 \%)$ se supone que están irregularmente (Fuente: Censo de 2001). Por otro lado, de las 20.000 personas que atraviesan anualmente de un modo ilegal la frontera sur española la mitad se desvanecen (Solé, Parella, Alarcón, Bergalli y Gilbert 2000: 139). La otra mitad es devuelta o expulsada.

14. Boschman y Fritsch (2009), usando información de 500 regiones de 7 países del centro-norte de Europa, concluyen que hay una íntima relación entre la proporción de bohemios y el volumen de clase creativa, que la presencia de bohemios es tan importante como las oportunidades de empleo y que el volumen de actividades de ocio y recreativas solo influye en la cantidad de clase creativa si la población bohemia disminuye

15. La población que podemos calificar de bohemia según las opciones que nos ofrecen los censos de $1991 \mathrm{y}$ del 2001 son discutibles. En el primero hay una sola categoría que encajaría con lo que Florida entiende como población bohemia. El problema es que solo tiene en cuenta a los escritores y profesionales del espectáculo e incluye, además, a los profesionales de los deportes que no encajan en lo que tradicionalmente se ha considerado como bohemio. En el censo del 2001 aparecen dos categorías en las que están las profesiones mencionadas antes pero a las que se añaden otras. Son los escritores y artistas de la creación e interpretación, por un lado, y los profesionales del mundo artístico, del espectáculo y de los deportes, por otro lado. Se deduce que la comparación es difícil. Además, nos hemos encontrado con la imposibilidad de utilizar las categorías de las últimas encuestas de población activa y poder así ampliar la comparación, pues, al tratarse de una encuesta, las categorías son más amplias y falta también la desagregación por provincias y comunidades autónomas. Pero es que tampoco hemos podido ampliar la comparación hacia atrás, pues en el censo de 1981 no aparecen desagregadas por provincias ni autonomías las dos categorías que encajaban con la noción de profesiones bohemias (por un lado escultores, pintores, fotógrafos y similares; por otro lado, profesionales de la música y espectáculos artísticos). En conjunto sumaban 49.634, apenas el 0,46 de la población. En fin, que apenas 1 de cada 200 nacionales era en aquella época bohemio.

16. Para encontrar esas "experiencias de autotrascendencia" (Joas 2002: 23 y ss.), en las que el yo se desborda y autoextravía "en pos de las fuerzas de la socialidad que lo constituyen y lo transforman" (P. 35), pues están en la base de la creatividad.

Boschma, R. A. y M. Fristch. 2009. Creative Class and regional Growth: Empirical Evidence from Seven European Countries". Economic Geography 4:391-423. http:// dx.doi.org/10.1111/j.1944-8287.2009.01048.x

Budd, W., Jr, N. Lovrich, J. C. Pierce y B. Chamberlain. 2008. "Cultural sources of variations in urban sustainability attributes". Cities 25:257-267. http://dx.doi.org/10.1016/j. cities.2008.05.001

Casanova, J. V. 2012. "Lo secular, las secularizaciones y los secularismos". Pp. 93-124 en Dialécticas de la postsecularidad. Pluralismo y corrientes de secularización, editado por I. Sánchez de la Yncera y M. Rodríguez Fouz. Barcelona: Anthropos.

Casares, P., P. Coto-Millán and V. Inglada. 2012. Creative economy and income level in Spanish regions. Revista Principios. Estudios de Economía Política 20: 87-104. 
Castoriadis, C. 1989. La institución imaginaria de la sociedad. Vol. 2. El imaginario social y la institución. Barcelona: Tusquets.

Christophers, B. 2008. "The BBC, the Creative Class and neoliberalism in the North of England". Environement and Planning A: 40:231-232N. http://dx.doi. org/10.1068/a4030

Csikszentmihalyi, M. 1996. Creativity: Flow and the Psychology of Discovery and Invention New York: Harper Perennial.

De Bono, E. 2006. El pensamiento lateral. Barcelona: Paidós.

De Certeau, M. 1990. L'invention du quotidien 1. Arts de faire. Paris: Gallimard.

Deleuze, G. y F. Guattari. 1986. Mil mesetas. Esquizofrenia y capitalismo. Valencia: Pre-Textos.

Donegan, M. y N. Lowe. 2008. "Inequality in the Creative City: Is Still a Place for Old-Fashioned Institutions?". Economic Development Quarterly 22:46-62. http:// dx.doi.org/10.1177/0891242407310722

Drucker, P. F. 1994. "The Age of Social Transformation". The Atlantic Monthly, Vol. 274, 5:53-80.

Feito, R. 1995. Estructura social contemporánea. Las clases sociales en los países industrializados. Madrid: Siglo $\mathrm{XXI}$

Fernández Esquinas, M. 2012. "Hacia un programa de investigación en sociología de la innovación”. Arbor 188:518. http://dx.doi.org/10.3989/arbor.2012.753n1001

Florida, R. 2002/2010. The Rise of the Creative Class. New York: Basic Books. PMCid:PMC1172185

Florida, R. 2005a. The Flight of the Creative Class. The New Global Competition for Talent. Nueva York: Harper Business, Harper Collins. PMCid:PMC2576447

Florida, R. 2005b. Cities and the Creative Class 2005. Londres: Routledge. PMCid:PMC2576447

Florida, R. y Ch. Mellander. 2009. "There goes the metro: how and why bohemians, artists and gays affect regional housing values". Journal of Economic Geography 10:167-188. http://dx.doi.org/10.1093/jeg/lbp022

Florida, R. y Tinagli. 2004. Europe in the Creative Age. Carnegie Mellon Software Industry Center, Alfred P. Sloan Foundation y Demos.

Fumagalli, A. 2010. Bioeconomía y capitalismo cognitivo. Hacia un nuevo paradigma de acumulación. Madrid: Los Libros de la Catarata.

García, J. L. 2012. "El discurso de la innovación en tela de juicio: tecnología, mercados y bienestar humano". Arbor 188:19-30. http://dx.doi.org/10.3989/ arbor.2012.753n1002

Gardner, H. 2010. Mentes creativas. Una anatomía de la creatividad. Barcelona: Paidós.

Goldthorpe, J. H. 2010. De la sociología. Números, narrativas e integración de la investigación y la teoría. Madrid: CIS. PMCid:PMC3014068

Houston, D., A. Finlay, R. Harrison y C. Mason. 2008. "Will attracting the Creative Class boost the Economic Growth in the Old Industrial Regions? A case Study of Scotland", Geografiska Annaler. Series B, Human Geography 90:133149. http://dx.doi.org/10.1111/j.1468-0467.2008.00283.x

Howkins, Jhon. 2005. The Creative Economy. How people make Money from ideas. Londres: Penguin Books.

Ingleghart, R. 1991. El cambio cultural en las sociedades industriales avanzadas. Madrid: CIS
Ingleghart, R. 2001. Modernización y postmodernización. Madrid: CIS.

Inglehart, R. y Ch. Welzel. 2006. Modernización, cambio cultural y democracia. La secuencia del desarrollo humano. Madrid: CIS.

Joas, H. 1996. The Creative of Action, Chicago: University of Chicago Press.

Joas, H. 2002. Creatividad, acción y valores. Hacia una sociología de la contingencia. México: Biblioteca de Signos.

Jünger, E. 1993. El trabajador. Dominio y figura. Barcelona: Tusquets.

Koestler, Arthur. 1975. The act of Creation. London: Picador.

Lahire, B. 2004. El hombre plural. Los resortes de la acción. Barcelona: Edicions Bellaterra.

Nancy, J. L. 2010. L'Adoration (Déconstruction du christianisme 2). Paris: Galilée. PMCid:PMC2897057

Navarro, C. J. y C. Mateos. 2010. La clase creativa en los municipios españoles. Propuesta de medición y análisis descriptivo. DT 04/10. Sevilla: Centro de Sociología y Políticas Locales, Universidad Pablo de Olavide.

Negrey, C. y S. Rausch. 2009. "Creativity gaps and gende gaps: women, men and place in the United States". Gender, Place and Culture 16:517-533. http://dx.doi. org/10.1080/09663690903148408

Noya Miranda, F. J. 2010. "Sociología de la creatividad". Pp. 19-40 en Musyca. Música, sociedad y creatividad artística, compilado por J. Noya, F. del Val y M. Pérez Colman. Madrid: Biblioteca Nueva.

Payne, A. y N. Philips. 2011. Desarrollo. Madrid: Alianza.

Peck, J. 2005. "Struggling with the Creative Class International". Journal of Urban and Regional Research 29:740-770. http://dx.doi.org/10.1111/j.1468-2427.2005.00620.x

Pohl, Th. 2008. "Distribution Patterns of the Creative Class in Hambourg: Oppenning to diversity as a driving forcé for socio-spacial differentation?". Erdkunde 62:317328. http://dx.doi.org/10.3112/erdkunde.2008.04.04

Qian, H. 2010. "Talent, creativity and regional economic performance: the case of China" Annals of Regional Science 45:133-156. http://dx.doi.org/10.1007/s00168-008-0282-3

Scott Allen, J. 2006. "Creative Cities: Conceptual Issues and Policy Questions". Journal of Urban Affairs 28:1-17. http://dx.doi.org/10.1111/j.0735-2166.2006.00256.x

Sen, A. 2000. Desarrollo y Libertad. Barcelona: Planeta.

Solé, C., S. Parella, A. Alarcón, U. Bergalli y F. Gilbert. 2000. "El impacto de la inmigración en la sociedad receptora". Revista Española de Investigaciones Sociológicas 90:131-157. http://dx.doi.org/10.2307/40184254

Tezanos, J. F. 2001. La sociedad dividida. Estructuras de clases en las sociedades tecnológicas. Madrid: Biblioteca Nueva.

Unesco 2005. Hacia las sociedades del conocimiento. París: Ediciones Unesco.

Uzzi, B. y J. Spiro. 2005. "Collaboration and creativity". American Journal of Sociology 76:262-272.

Villaverde Rico, M. J. y J. C. Laursan. 2011. Forjadores de la tolerancia. Madrid: Tecnos.

Wrigth, Erik Olin 1983. Clase, crisis y Estado. Madrid: Siglo $\mathrm{XXI}$. 
JOSÉ ANGEL BERGUA AMORES. Profesor titular en la Facultad de Economía y Empresa de la Universidad de Zaragoza y acreditado como catedrático. Sus principales líneas de investigación son la creatividad, las modas, los jóvenes, los conflictos medioambientales, la crisis de la occidentalidad y distintas cuestiones teóricas relacionadas con la actividad social instituyente (el desorden, lo imaginario, las anamnesis, lo sagrado, la gente, etc.). Entre otros libros ha publicado La gente contra la sociedad. Impacto sociocultural de un divertimento juvenil (2002), Patologías de la Modernidad (2005), Lo social instituyente (2007), Estilos de la investigación social (2011), Anarquías. Ámbitos no jerárquicos de lo social (2013) y Postpolítica. Elogio del gentío (2015).

JUAN MIGUEL BÁEZ. Doctor en Economía Aplicada por la ULPGC. Profesor Contratado Doctor del Departamento de Dirección y Organización de Empresas de la Universidad de Zaragoza. Sus líneas de investigación son la economía creativa, la desigualdad económica y la ayuda al desarrollo.

DAVID PAC SALAS. Profesor Contratado Doctor del Departamento de Psicología y Sociología en la Universidad de Zaragoza. Licenciado en Ciencias Políticas y Sociología
(Universidad Autónoma de Barcelona) y Diplomado Universitario Graduado Social y Doctor en Sociología (Universidad de Zaragoza). En la actualidad trabaja sobre ocupaciones creativas, consumo y cuestiones vinculadas a la sociología política. Sobre estas temáticas, ha publicado artículos en Creativity Research Journal y Revista Política y Gobierno. Es coautor (junto con José Angel Bergua, Juan Miguel Báez y Enrique Carretero) de Creatividad: números e imaginarios, publicada en el Centro de Investigaciones Sociológicas (2016). Acaba de regresar de una estancia de investigación de tres meses, en el Instituto de Investigaciones Gino Germani de la Universidad de Buenos Aires, donde ha investigado las relaciones entre creatividad y estilos de vida. Es Decano del Colegio de Doctores y Licenciados en Ciencias Políticas y Sociología de Aragón.

CECILIA SERRANO MARTíNEZ. Doctora en Sociología por la Universidad de Zaragoza y Premio Extraordinario de Doctorado. Ha escrito e investigado sobre creatividad, juventud y el mundo del arte. Compagina su actividad sociológica con el teatro. En la actualidad participa en una IAP que se propone realizar una intervención creativa en la ciudad de Zaragoza. 